\title{
THE EXISTENCE OF LOCAL SOLUTIONS FOR THE COMPRESSIBLE NAVIER-STOKES EQUATIONS WITH THE DENSITY-DEPENDENT VISCOSITIES*
}

\author{
PEIXIN ZHANG ${ }^{\dagger}$ AND JUNNING ZHAO $\ddagger$
}

\begin{abstract}
In this paper, we consider the isentropic compressible Navier-Stokes equations with density-dependent viscosities. We prove the local existence of the classical solutions, where the initial density is allowed to vanish.
\end{abstract} lution.

Key words. Compressible Navier-Stokes equations, density-dependent viscosities, classical so-

AMS subject classifications. $76 \mathrm{~N} 10$.

\section{Introduction}

The motion of a compressible viscous barotropic fluid in a bounded domain $\Omega$ in $\mathbb{R}^{3}$ can be described by the compressible Navier-Stokes equations

$$
\left\{\begin{array}{l}
\rho_{t}+\operatorname{div}(\rho u)=0, \\
(\rho u)_{t}+\operatorname{div}(\rho u \otimes u)+L u+\nabla P(\rho)=0, \\
L u=-\operatorname{div}(2 \mu \mathcal{D} u)-\nabla(\lambda \operatorname{div} u),
\end{array}\right.
$$

and the boundary conditions

$$
\begin{cases}\left.(\rho, u)\right|_{t=0}= & \left(\rho_{0}, u_{0}\right), \text { in } \Omega, \\ u=0, & \text { on }(0, T) \times \partial \Omega,\end{cases}
$$

where $\rho, u=\left(u^{1}, u^{2}, u^{3}\right)$ and $P=A \rho^{\gamma}(A>0, \gamma>1)$ are the fluid density, velocity, and pressure respectively, $\mathcal{D} u=\frac{1}{2}\left(\nabla u+{ }^{t} \nabla u\right)$ is the strain tensor, and $\Omega \subset \mathbb{R}^{3}$ is a bounded domain with smooth boundary $\partial \Omega$. The viscosity coefficients $\mu=\mu(\rho)$ and $\lambda=\lambda(\rho)$ are the functions of the density $\rho$. Here we consider the following two cases:

$$
\lambda(\cdot) \in C^{3}[0, \infty), \mu(\cdot) \in C^{3}[0, \infty) \text { and } \mu(\cdot) \geq \mu_{0}>0,2 \mu+3 \lambda \geq 0,
$$

or

$$
\lambda(\cdot) \in C^{3}[0, \infty), \mu(\cdot)=A_{1} \rho^{\alpha}, \quad 2 \mu+3 \lambda \geq 0,
$$

where $\alpha$ is an arbitrary real number and $\mu_{0}, A_{1}$ are positive constants.

There are huge literatures on the existence and behavior of solutions for compressible Navier-Stokes equations. For the case where that the viscosity coefficients are constants and the initial density $\rho_{0}>0$, the one-dimensional problem has been studied extensively by many people; see [11, 18, 27, 28] and the references therein. For the multi-dimensional case, the local existence and uniqueness of classical solutions can be found in $[26,29]$. The global classical solutions were obtained by

${ }^{*}$ Received: January 22, 2013; accepted (in revised form): September 21, 2013. Communicated by Zhouping Xin.

${ }^{\dagger}$ School of Mathematical Sciences, Huaqiao University, Quanzhou, 362021, P.R. China \& School of Mathematical Sciences, Xiamen University, Xiamen, 361005, P.R. China (zhpx@hqu.edu.cn).

${ }^{\ddagger}$ School of Mathematical Sciences, Xiamen University, Xiamen, 361005, P.R. China (jnzhao@xmu.edu.cn). 
Matsumura-Nishida [24] for initial data close to a non vacuum equilibrium in some Sobolev space $H^{s}$. Later, Hoff $[12,13]$ studied the problem for discontinuous initial data. For a general initial density $\rho_{0} \geq 0$, the existence of weak solutions is due to Lions [21] (see also Feireisl [7]), the local existence of strong/classical solutions were obtained by $[3,4,5]$ and the global classical solution was studied in [14].

From physical considerations, the viscosity coefficients are functions of the temperature. If we consider the case of isentropic fluids, this dependence is reduced to the dependence on the density (see [10]). For the case that the viscosity coefficients are functions of density or temperature, the one-dimensional problem has been studied widely; the local existence theorems were obtained by Makino in [23] and Liu-XinYang in [22], and the global existence was studied in [6, 8, 9, 16, 31, 32, 33]. For the multi-dimensional problem, if the initial density is away from vacuum and $\mu \geq \mu_{1}>0$ ( $\mu_{1}$ is a constant), the local existence of strong solutions was obtained by Valli in [30], the global strong/classical solutions were obtained in [17,34] under the assumption that the initial data are small in some Sobolev space $H^{s}$. When the initial density is allowed to vanish, the global weak solutions were studied in $[1,25]$.

The purpose of our paper is to prove the local existence of classical solutions when $\mu>0$ and the initial density $\rho_{0} \geq 0$, or $\mu=A_{1} \rho^{\alpha}, 2 \mu+3 \lambda \geq 0, \rho_{0}>0$. Because the initial density is allowed to vanish, the equations have singularity, so some methods used in previous papers cannot be applied to our case. The main difficulties arise from the fact that the viscous coefficients $\mu, \lambda$ are functions of $\rho$ and that equation $(1.1)_{2}$ has a singularity. In this paper, we have to deal with the higher order estimates of the density. Moreover some ideas in $[4,5]$ are used.

Before stating the main results, we explain the notations and conventions used throughout this paper. We denote

$$
\int f d x=\int_{\Omega} f d x
$$

For $1<r<\infty$ and $k$ is a positive integer, the standard homogeneous and inhomogeneous Sobolev spaces as follows:

$$
\left\{\begin{array}{l}
L^{r}=L^{r}(\Omega), D^{k, r}=\left\{u \in L_{\mathrm{loc}}^{1}(\Omega) \mid\left\|\nabla^{k} u\right\|_{L^{r}}<\infty\right\},\|u\|_{D^{k, r}}:=\left\|\nabla^{k} u\right\|_{L^{r}}, \\
W^{k, r}=L^{r} \cap D^{k, r}, H^{k}=W^{k, 2}, D^{k}=D^{k, 2}, H_{0}^{1}=\left\{u \in H^{1} \mid u=0 \text { on } \partial \Omega\right\}, \\
D^{1}=\left\{u \in L^{6} \mid\|\nabla u\|_{L^{2}}<\infty\right\} .
\end{array}\right.
$$

The main results in this paper can be stated as follows.

Theorem 1.1. Assume that $\mu, \lambda$ satisfy (1.3) and that the initial data $\left(\rho_{0}, u_{0}\right)$ satisfy

$$
0 \leq \rho_{0} \in H^{3}, P\left(\rho_{0}\right) \in H^{3}, \quad u_{0} \in H_{0}^{1} \cap H^{3},
$$

and the compatibility condition

$$
-\operatorname{div}\left(2 \mu\left(\rho_{0}\right) \mathcal{D} u_{0}\right)-\nabla\left(\lambda\left(\rho_{0}\right) \operatorname{divu}_{0}\right)+\nabla P\left(\rho_{0}\right)=\rho_{0} g,
$$

for some $g \in H_{0}^{1}$. Then there exists a time $T_{*}>0$ such that (1.1)-(1.2) has a unique 
classical solution $(\rho, u)$ on $\Omega \times\left(0, T_{*}\right)$ which satisfies

$$
\left\{\begin{array}{l}
\rho \in C\left(\left[0, T_{*}\right] ; H^{3}\right), \quad \rho_{t} \in C\left(\left[0, T_{*}\right] ; H^{2}\right), \\
\rho_{t t} \in L^{\infty}\left(0, T_{*} ; L^{2}\right) \cap L^{2}\left(0, T_{*} ; H^{1}\right), \\
u \in C\left(\left[0, T_{*}\right] ; H_{0}^{1} \cap H^{3}\right) \cap L^{2}\left(0, T_{*} ; H^{4}\right) \cap L^{\infty}\left(\tau, T_{*} ; H^{4}\right), \\
u_{t} \in L^{\infty}\left(0, T_{*} ; H_{0}^{1}\right) \cap L^{2}\left(0, T_{*} ; H^{2}\right), \sqrt{\rho} u_{t} \in L^{\infty}\left(0, T_{*} ; L^{2}\right), \\
\sqrt{\rho} u_{t t} \in L^{2}\left(0, T_{*} ; L^{2}\right), \sqrt{\rho} u_{t t t} \in L^{2}\left(\tau, T_{*} ; L^{2}\right), \\
u_{t t} \in L^{\infty}\left(\tau, T_{*} ; H^{1}\right) \cap L^{2}\left(\tau, T_{*} ; H^{2}\right),
\end{array}\right.
$$

where $\tau \in\left(0, T_{*}\right)$ is any positive constant.

We can also prove the following.

Theorem 1.2. Assume that $\mu, \lambda$ satisfy (1.4) and $0<\rho \leq \rho_{0} \in H^{3}$, $\rho$ is a constant, and $u_{0} \in H_{0}^{1} \cap H^{3}$. Then there exists a time $T_{*}>0$ and a unique classical solution $(\rho, u)$ satisfying the regularity properties in (1.7).

By virtue of Theorem 1.1, we also can prove the existence of the local solution for the Cauchy problem of (1.1). Consider the Cauchy problem for the equation (1.1) with the far field behavior

$$
u(x, t) \rightarrow 0, \rho(x, t) \rightarrow 0 \text { as }|x| \rightarrow \infty
$$

and initial data

$$
\left.(\rho, u)\right|_{t=0}=\left(\rho_{0}, u_{0}\right), \quad x \in \mathbb{R}^{3} .
$$

Theorem 1.3. Assume that $\mu, \lambda$ satisfy (1.3) and that the initial data $\left(\rho_{0}, u_{0}\right)$ satisfy

$$
0 \leq \rho_{0} \in H^{3}, P\left(\rho_{0}\right) \in H^{3}, \quad u_{0} \in D^{1} \cap D^{3},
$$

and the compatibility condition

$$
-\operatorname{div}\left(2 \mu\left(\rho_{0}\right) \mathcal{D} u_{0}\right)-\nabla\left(\lambda\left(\rho_{0}\right) \operatorname{divu}_{0}\right)+\nabla P\left(\rho_{0}\right)=\rho_{0} g,
$$

for some $g \in D^{1}$ with $\rho_{0}^{1 / 2} g \in L^{2}$. Then there exists a time $T_{*}>0$ such that (1.1),(1.8),(1.9) has a unique classical solution $(\rho, u)$ on $\mathbb{R}^{3} \times\left(0, T_{*}\right)$ which satisfies

$$
\left\{\begin{array}{l}
\rho \in C\left(\left[0, T_{*}\right] ; H^{3}\right), \quad \rho_{t} \in C\left(\left[0, T_{*}\right] ; H^{2}\right), \\
\rho_{t t} \in L^{\infty}\left(0, T_{*} ; L^{2}\right) \cap L^{2}\left(0, T_{*} ; H^{1}\right), \\
u \in C\left(\left[0, T_{*}\right] ; D^{1} \cap D^{3}\right) \cap L^{2}\left(0, T_{*} ; D^{4}\right) \cap L^{\infty}\left(\tau, T_{*} ; D^{4}\right), \\
u_{t} \in L^{\infty}\left(0, T_{*} ; D^{1}\right) \cap L^{2}\left(0, T_{*} ; D^{2}\right), \sqrt{\rho} u_{t} \in L^{\infty}\left(0, T_{*} ; L^{2}\right), \\
\sqrt{\rho} u_{t t} \in L^{2}\left(0, T_{*} ; L^{2}\right), \sqrt{\rho} u_{t t t} \in L^{2}\left(\tau, T_{*} ; L^{2}\right), \\
u_{t t} \in L^{\infty}\left(\tau, T_{*} ; D^{1}\right) \cap L^{2}\left(\tau, T_{*} ; D^{2}\right),
\end{array}\right.
$$

where $\tau \in\left(0, T_{*}\right)$ is any positive constant.

The rest of this paper is organized as follows. In Section 2, we give some elementary facts, and then study the linearized problem of (1.1) and prove some existence and regularity results for a linear transport equation and a linear parabolic system. In Section 3, we first construct an approximate solution to the Navier-Stokes equations (1.1)-(1.2), then we derive some uniform estimates in higher norms, which implies the local existence of classical solutions of (1.1)-(1.2). Theorem 1.2 and Theorem 1.3 are proved in Section 4. 


\section{Preliminaries}

In this section, we will give some elementary facts which will be used later.

LEMMA 2.1. (Korn's inequality [15]) Let $\Omega$ is a bounded domain in $\mathbb{R}^{3}$ with smooth boundary. Assume that $\mu, \lambda$ satisfy (1.3), $u \in H_{0}^{1} \cap H^{2}$. Then there exists a positive constant $C=C(\Omega, \mu, \lambda)$ such that

$$
\int\left(2 \mu|\mathcal{D} u|^{2}+\lambda(\text { divu })^{2}\right) d x \geq C \int|\nabla u|^{2} d x
$$

Lemma 2.2. ([2]) Let $\Omega$ be a bounded domain of $\mathbb{R}^{n}$ and suppose $\partial \Omega$ is $C^{1}$. Assume $1 \leq p<n$, and $u \in W^{1, p}(\Omega)$. Then $u \in L^{p^{*}}(\Omega)$ with the estimate

$$
\|u\|_{L^{p^{*}}} \leq C\|u\|_{W^{1, p}}, \quad p^{*} \in\left[1, \frac{n p}{n-p}\right] .
$$

Moreover if $p>n$, we have

$$
\|u\|_{L^{\infty}} \leq C\|u\|_{W^{1, p}}
$$

where $C$ depends only on $p, n$, and $\Omega$.

Consider the elliptic system

$$
\left\{\begin{array}{l}
\sum_{j=1}^{N} \sum_{\alpha \beta=1}^{n} D_{\alpha}\left(A_{i j}^{\alpha \beta}(x) D_{\beta} u^{j}\right)=f_{i}, \quad i=1, \ldots, N \\
u=0, \quad x \in \partial \Omega
\end{array}\right.
$$

where $\Omega \subseteq \mathbb{R}^{n}$ is a smooth bounded domain, and

$$
A_{i j}^{\alpha \beta}(x) \xi_{\alpha}^{i} \xi_{\beta}^{j} \geq C_{0}|\xi|^{2}, C_{0}>0, A_{i j}^{\alpha \beta}(x) \in L^{\infty}, \forall \xi \in R^{n} .
$$

The following lemma can be found in [2] and [20].

Lemma 2.3. Assume that $f_{i} \in L^{2},\left|\nabla A_{i j}^{\alpha \beta}(x)\right||\nabla u| \in L^{2}$. Then the solution $u$ of (2.4) satisfies $u \in H^{2}$ and

$$
\int_{\Omega}\left|\nabla^{2} u\right|^{2} d x \leq C \int_{\Omega}\left(|\nabla u|^{2}+\left|\nabla A_{i j}^{\alpha \beta}(x)\right|^{2}|\nabla u|^{2}+\sum_{i=1}^{N}\left|f_{i}\right|^{2}\right) d x .
$$

We now study the linearized problem of (1.1). Consider the following linearized system:

$$
\left\{\begin{array}{l}
\rho_{t}+\operatorname{div}(\rho v)=0 \\
\rho u_{t}+\rho v \cdot u+L u+\nabla P(\rho)=0
\end{array}\right.
$$

where $L u=-\operatorname{div}(2 \mu \mathcal{D} u)-\nabla(\lambda \operatorname{div} u), P=A \rho^{\gamma}$ and $v$ is a known vector field.

Lemma 2.4. Assume that $\lambda, \mu$ satisfy (1.3) and $\left(\rho_{0}, u_{0}\right)$ satisfy the regularity conditions

$$
\delta \leq \rho_{0} \in H^{3} \quad \text { for some } \delta>0, \quad u_{0} \in H_{0}^{1} \cap H^{3},
$$


and that $v$ satisfies the regularity conditions

$$
\begin{aligned}
& v \in C\left([0, T] ; H_{0}^{1} \cap H^{3}\right) \cap L^{2}\left(0, T ; H^{4}\right), \\
& v_{t} \in C\left([0, T] ; H_{0}^{1}\right) \cap L^{2}\left(0, T ; H^{2}\right) .
\end{aligned}
$$

Then there exists a unique classical solution $(\rho, u)$ to the problem (2.5), (1.2) such that

$$
\left\{\begin{array}{l}
\rho \in C\left([0, T] ; H^{3}\right), \quad \rho_{t} \in C\left([0, T] ; H^{2}\right), \\
\rho_{t t} \in L^{\infty}\left(0, T ; L^{2}\right) \cap L^{2}\left(0, T ; H^{1}\right), \\
u \in C\left([0, T] ; H_{0}^{1} \cap H^{3}\right) \cap L^{2}\left(0, T ; H^{4}\right) \cap L^{\infty}\left(\tau, T ; H^{4}\right), \\
u_{t} \in L^{\infty}\left(0, T ; H_{0}^{1}\right) \cap L^{2}\left(0, T ; H^{2}\right), \sqrt{\rho} u_{t} \in L^{\infty}\left(0, T ; L^{2}\right), \\
\sqrt{\rho} u_{t t} \in L^{2}\left(0, T ; L^{2}\right), \sqrt{\rho} u_{t t t} \in L^{2}\left(\tau, T ; L^{2}\right) \\
u_{t t} \in L^{\infty}\left(\tau, T ; H^{1}\right) \cap L^{2}\left(\tau, T ; H^{2}\right), \\
\rho>0 \text { on }[0, T] \times \bar{\Omega}
\end{array}\right.
$$

where $\tau \in(0, T)$ is any positive constant.

Proof. Clearly, the equation $(2.5)_{1}$ has a unique solution $\rho$, which can be expressed by

$$
\rho(t, x)=\rho_{0}(U(0, t, x)) \exp \left(-\int_{0}^{t} \operatorname{div} v(s, U(s, t, x)) d s\right),
$$

where $U=U(t, s, x)$ is the solution of

$$
\begin{cases}\frac{\partial}{\partial t} U(t, s, x)=v(t, U(t, s, x)), & 0 \leq t \leq T \\ U(s, s, x)=x, & 0 \leq s \leq T, \quad x \in \Omega .\end{cases}
$$

Using (2.7) and Sobolev's inequality, we have

$$
\rho(t, x) \geq \delta \exp \left(-\int_{0}^{T}\|\nabla v\|_{H^{2}} d t\right)>0,
$$

for $(t, x) \in[0, T] \times \bar{\Omega}$.

We now prove

$$
\begin{aligned}
\sup _{0 \leq t \leq T}\left(\|\rho\|_{H^{3}}+\|P\|_{H^{3}}+\left\|\rho_{t}\right\|_{H^{2}}\right. & \left.+\left\|P_{t}\right\|_{H^{2}}+\left\|\rho_{t t}\right\|_{L^{2}}+\left\|P_{t t}\right\|_{L^{2}}\right) \\
& +\int_{0}^{T}\left(\left\|\nabla \rho_{t t}\right\|_{L^{2}}^{2}+\left\|\nabla P_{t t}\right\|_{L^{2}}^{2}\right) d t \leq \tilde{C},
\end{aligned}
$$

where and in rest of the proof of this lemma, we denote by $\tilde{C}$ a generic positive constant depending only on the norm of $v,\left\|\rho_{0}\right\|_{H^{3}}$, and $T$, but independent of $\delta$.

Multiplying the equation (2.5) 1 by $\rho$ and integrating (by parts) over $\Omega$, we obtain

$$
\frac{d}{d t} \int|\rho|^{2} d x \leq C \int|\nabla v \| \rho|^{2} d x \text {. }
$$

Sobolev's inequality thus yields

$$
\frac{d}{d t}\|\rho\|_{L^{2}}^{2} \leq C\|\nabla v\|_{L^{\infty}}\|\rho\|_{L^{2}}^{2} \leq C\|v\|_{H^{3}}\|\rho\|_{L^{2}}^{2} .
$$


Differentiating $(2.5)_{1}$ with respect to $x_{j}$, multiplying by $\partial_{j} \rho$ and then integrating over $\Omega$, we have

$$
\begin{aligned}
\frac{d}{d t} \int\left|\partial_{j} \rho\right|^{2} d x & \leq C \int\left(\left|\nabla v\left\|\left.\nabla \rho\right|^{2}+|\rho|\left|\nabla \rho \| \nabla^{2} v\right|\right) d x\right.\right. \\
& \leq C\left(\|v\|_{H^{3}}\|\rho\|_{H^{1}}^{2}+\|\rho\|_{L^{3}}\|\nabla \rho\|_{L^{2}}\left\|\nabla^{2} v\right\|_{L^{6}}\right) .
\end{aligned}
$$

Using Sobolev's inequality, we have

$$
\frac{d}{d t}\left\|\partial_{j} \rho\right\|_{L^{2}}^{2} \leq C\|v\|_{H^{3}}\|\rho\|_{H^{1}}^{2} .
$$

Similarly, differentiating $(2.5)_{1}$ with respect to $x_{j}$ and $x_{i}$, multiplying by $\partial_{j} \partial_{i} \rho, 1 \leq i, j \leq 3$, and then integrating the resulting equation over $\Omega$, we have

$$
\begin{aligned}
\frac{d}{d t} \int\left|\partial_{j} \partial_{i} \rho\right|^{2} d x \leq & C \int\left(\left|\nabla v\left\|\left.\nabla^{2} \rho\right|^{2}+\left|\nabla^{2} v\|\nabla \rho\| \nabla^{2} \rho\right|+\rho\left|\nabla^{2} \rho \| \nabla^{3} v\right|\right) d x\right.\right. \\
\leq & C\left(\|\nabla v\|_{L^{\infty}}\left\|\nabla^{2} \rho\right\|_{L^{2}}^{2}+\|\nabla \rho\|_{L^{6}}\left\|\nabla^{2} v\right\|_{L^{3}}\left\|\nabla^{2} \rho\right\|_{L^{2}}\right. \\
& \left.\quad+\|\rho\|_{L^{\infty}}\left\|\nabla^{3} v\right\|_{L^{2}}\left\|\nabla^{2} \rho\right\|_{L^{2}}\right) \\
\leq & C\|v\|_{H^{3}}\|\rho\|_{H^{2}}^{2} .
\end{aligned}
$$

Similarly, we have

$$
\begin{gathered}
\frac{d}{d t} \int\left|\nabla^{3} \rho\right|^{2} d x \leq C \int\left(\left|\nabla^{3} \rho\right|^{2}|\nabla v|+\left|\nabla^{3} \rho \| \nabla^{2} \rho\right|\left|\nabla^{2} v\right|\right. \\
\left.+\left|\nabla^{3} \rho\|\nabla \rho\| \nabla^{3} v\right|+\rho\left|\nabla^{3} \rho \| \nabla^{4} v\right|\right) d x \\
\leq C\|v\|_{H^{3}}\left\|\nabla^{3} \rho\right\|_{L^{2}}^{2}+C\|v\|_{H^{4}}\left(\left\|\nabla^{3} \rho\right\|_{L^{2}}^{2}+\left\|\nabla^{2} \rho\right\|_{L^{2}}^{2}\right) \\
\quad+C\|\nabla \rho\|_{L^{3}}\left\|\nabla^{3} \rho\right\|_{L^{2}}\left\|\nabla^{3} v\right\|_{L^{6}}+C\|\rho\|_{L^{\infty}}\left\|\nabla^{3} \rho\right\|_{L^{2}}\left\|\nabla^{4} v\right\|_{L^{2}} \\
\leq C\|v\|_{H^{4}}\|\rho\|_{H^{3}}^{2} .
\end{gathered}
$$

Combining (2.9)-(2.12), we get

$$
\sup _{0 \leq t \leq T}\|\rho\|_{H^{3}} \leq \tilde{C} .
$$

Because $\rho_{t}=-v \cdot \nabla \rho-\rho \operatorname{div} v=-v^{k} \partial_{k} \rho-\rho \partial_{k} v^{k}$ and

$$
\begin{gathered}
\partial_{i} \partial_{j} \rho_{t}=-v^{k} \partial_{i} \partial_{j} \partial_{k} \rho-\partial_{i} v^{k} \partial_{j} \partial_{k} \rho-\partial_{j} v^{k} \partial_{i} \partial_{k} \rho-\partial_{i} \partial_{j} v^{k} \partial_{k} \rho-\partial_{i} \partial_{j} \rho \partial_{k} v^{k} \\
-\partial_{i} \rho \partial_{j} \partial_{k} v^{k}-\partial_{j} \rho \partial_{i} \partial_{k} v^{k}-\rho \partial_{i} \partial_{j} \partial_{k} v^{k}
\end{gathered}
$$

then we have

$$
\sup _{0 \leq t \leq T}\left\|\rho_{t}\right\|_{L^{2}} \leq C \sup _{0 \leq t \leq T}\left(\|v\|_{L^{3}}\|\nabla \rho\|_{L^{6}}+\|\rho\|_{L^{3}}\|\operatorname{div} v\|_{L^{6}}\right) \leq \tilde{C}
$$

and

$$
\begin{array}{cc}
\sup _{0 \leq t \leq T}\left\|\nabla^{2} \rho_{t}\right\|_{L^{2}} \leq C \sup _{0 \leq t \leq T}\left(\|v\|_{L^{\infty}}\left\|\nabla^{3} \rho\right\|_{L^{2}}+\|\nabla v\|_{L^{3}}\left\|\nabla^{2} \rho\right\|_{L^{6}}\right. \\
\\
\left.\quad+\left\|\nabla^{2} v\right\|_{L^{3}}\|\nabla \rho\|_{L^{6}}+\|\rho\|_{L^{\infty}}\left\|\nabla^{3} v\right\|_{L^{2}}\right) \\
\leq \tilde{C}
\end{array}
$$


Differentiating $(2.5)_{1}$ with respect to $t$, we have

$$
\rho_{t t}+\rho_{t} \operatorname{div} v+\rho \operatorname{div} v_{t}+\nabla \rho_{t} \cdot v+\nabla \rho \cdot v_{t}=0
$$

Thus

$$
\begin{aligned}
& \sup _{0 \leq t \leq T}\left\|\rho_{t t}\right\|_{L^{2}} \leq C \sup _{0 \leq t \leq T}\left(\left\|\rho_{t}\right\|_{L^{6}}\|\nabla v\|_{L^{3}}+\|\rho\|_{L^{\infty}}\left\|\nabla v_{t}\right\|_{L^{2}}\right. \\
& \\
& \leq \tilde{C},\left.+\left\|\nabla \rho_{t}\right\|_{L^{2}}\|v\|_{L^{\infty}}+\|\nabla \rho\|_{L^{3}}\left\|v_{t}\right\|_{L^{6}}\right) \\
&
\end{aligned}
$$

and

$$
\begin{aligned}
\int_{0}^{T}\left\|\nabla \rho_{t t}\right\|_{L^{2}}^{2} d t \leq & C \int_{0}^{T}\left(\left\|\rho_{t}\right\|_{L^{\infty}}\left\|\nabla^{2} v\right\|_{L^{2}}+\|\rho\|_{L^{\infty}}\left\|\nabla^{2} v_{t}\right\|_{L^{2}}\right. \\
& +\|\nabla \rho\|_{L^{\infty}}\left\|\nabla v_{t}\right\|_{L^{2}}+\left\|\nabla \rho_{t}\right\|_{L^{2}}\|\nabla v\|_{L^{\infty}}+\left\|\nabla^{2} \rho_{t}\right\|_{L^{2}}\|v\|_{L^{\infty}} \\
& \left.+\left\|\nabla^{2} \rho\right\|_{L^{3}}\left\|v_{t}\right\|_{L^{6}}+\left\|\nabla^{2} \rho_{t}\right\|_{L^{2}}\|v\|_{L^{\infty}}\right)^{2} d t \leq \tilde{C}
\end{aligned}
$$

Note that $P=A \rho^{\gamma}$ satisfies

$$
P_{t}+v \cdot \nabla P+\gamma P \operatorname{div} v=0
$$

Similarly to the above proof, we can prove

$$
\sup _{0 \leq t \leq T}\left(\|P\|_{H^{3}}+\left\|P_{t}\right\|_{H^{2}}+\left\|P_{t t}\right\|_{L^{2}}\right)+\int_{0}^{T}\left\|\nabla P_{t t}\right\|_{L^{2}}^{2} d t \leq \tilde{C} .
$$

Hence (2.8) is proved. Note that $(2.5)_{2}$ can be written as a linear parabolic system

$$
u_{t}+v \cdot \nabla u+\rho^{-1} L u=F,
$$

where $F=-\rho^{-1} \nabla P . \quad(2.8)$ implies that $F \in L^{\infty}\left(0, T ; H^{2}\right)$ and $F_{t} \in C\left([0, T] ; H^{1}\right) \cap$ $H^{1}\left(0, T ; L^{2}\right)$. Hence the existence and regularity $(2.6)$ of the solution of $(2.13)$ follow from the parabolic system theory (see [19]).

\section{Proof of Theorem 1.1.}

Without loss of generality, let $\rho_{0} \in C^{3}(\bar{\Omega})$ and $\rho_{0 \delta}=\rho_{0}+\delta$. From $P\left(\rho_{0}\right) \in H^{3}$, we have $P\left(\rho_{0 \delta}\right) \rightarrow P\left(\rho_{0}\right)$ in $H^{3}$ as $\delta \rightarrow 0$. Let $u_{0 \delta} \in H_{0}^{1} \cap H^{3}$ be a unique solution to the following elliptic boundary value problem:

$$
L_{\delta} u_{0 \delta}=-\operatorname{div}\left(2 \mu\left(\rho_{0 \delta}\right) \mathcal{D} u_{0 \delta}\right)-\nabla\left(\lambda\left(\rho_{0 \delta}\right) \operatorname{div} u_{0 \delta}\right)=F_{0 \delta} \text { in } \Omega \text { and } u_{0 \delta}=0 \text { on } \partial \Omega \text {, }
$$

where

$$
F_{0 \delta}=-\nabla P\left(\rho_{0 \delta}\right)+\rho_{0 \delta} g
$$

We will show that

$$
\left\{\begin{array}{l}
u_{0 \delta} \rightarrow u_{0} \text { in } H^{2} \text { as } \delta \rightarrow 0, \\
D^{3} u_{0 \delta} \rightarrow D^{3} u_{0} \text { in } L^{2} \text { as } \delta \rightarrow 0, \text { and }\left\|D^{3} u_{0 \delta}\right\|_{L^{2}} \leq C\left\|D^{3} u_{0}\right\|_{L^{2}}+C,
\end{array}\right.
$$

According to the theory of elliptic systems, we have

$$
\left\|u_{0 \delta}\right\|_{H^{3}} \leq C\left\|F_{0 \delta}\right\|_{H^{1}}+C\left\|\nabla \rho_{0}\right\|_{H^{2}} \leq C_{1},
$$


where $\mathrm{C}, C_{1}$ are the constants independent of $\delta, \Omega$, and $\nabla P\left(\rho_{0 \delta}\right) \rightarrow \nabla P\left(\rho_{0}\right)$ in $H^{1}$ is used. This means that there exists a sequence $\delta_{j}, \delta_{j} \rightarrow 0$ such that $\left\{u_{0 \delta_{j}}\right\}$ converges strongly in $H^{2}$ to a limit $\bar{u}_{0} \in H^{2}$ and $\left\{D^{3} u_{0 \delta_{j}}\right\}$ converges weakly in $L^{2}$ to $D^{3} \bar{u}_{0}$. Letting $\delta_{j} \rightarrow 0$ in (3.1), we get

$$
L \bar{u}_{0}=-\operatorname{div}\left(2 \mu\left(\rho_{0}\right) \mathcal{D} \bar{u}_{0}\right)-\nabla\left(\lambda\left(\rho_{0}\right) \operatorname{div} \bar{u}_{0}\right)=-\nabla P\left(\rho_{0}\right)+\rho_{0} g=F_{0},
$$

a.e. in $\Omega$. Note that $L u_{0}=F_{0}$. The uniqueness of solutions of elliptic problems implies $\bar{u}_{0}=u_{0}$. Hence $(3.2)$ holds.

To prove the existence of solutions of (1.1)-(1.2), we construct approximate solutions inductively as follows:

(i) first define $u^{0}=0$,

(ii) assume that $u^{k-1}$ was defined for $k \geq 1$, let $\left(\rho^{k}, u^{k}\right)$ be the unique global classical solution to the linearized problem (2.5) and (1.2) with $v$ replaced by $u^{k-1}$, i.e.

$$
\begin{gathered}
\rho_{t}^{k}+u^{k-1} \cdot \nabla \rho^{k}+\rho^{k} \operatorname{div} u^{k-1}=0, \quad \text { in } \quad(0, T) \times \Omega, \\
\rho^{k} u_{t}^{k}+\rho^{k} u^{k-1} \cdot \nabla u^{k}+L u^{k}+\nabla P^{k}=0, \quad \text { in } \quad(0, T) \times \Omega, \\
\left.\rho^{k}\right|_{t=0}=\rho_{0 \delta},\left.\quad u^{k}\right|_{t=0}=u_{0 \delta}, \quad \text { in } \Omega, \quad u^{k}=0, \quad \text { on }(0, T) \times \partial \Omega,
\end{gathered}
$$

where

$$
L u^{k}=-\operatorname{div}\left(2 \mu\left(\rho^{k}\right) \mathcal{D} u^{k}\right)-\nabla\left(\lambda\left(\rho^{k}\right) \operatorname{div} u^{k}\right), P^{k}=A\left(\rho^{k}\right)^{\gamma} .
$$

According to Lemma 2.4, the linearized problem (3.3)-(3.5) has a global classical solution $\left(\rho^{k}, u^{k}\right)$ with the regularity $(2.6)$.

Next, we show that the approximate solutions satisfy some uniform estimates to $k$ and $\delta$, and converge to a local classical solution of (1.1)-(1.2).

We introduce a function $\Phi_{K}(t)$ ( $K$ is a fixed positive integer) defined by

$$
\Phi_{K}(t)=\max _{1 \leq k \leq K} \sup _{0 \leq s \leq t}\left(1+\left\|\rho^{k}(s)\right\|_{H^{3}}+\left\|u^{k}(s)\right\|_{H^{3}}+\left\|\sqrt{\rho^{k}} u_{t}^{k}(s)\right\|_{L^{2}}+\left\|u_{t}^{k}(s)\right\|_{H^{1}}\right) .
$$

Then we prove that $\Phi_{K}$ is locally bounded.

To simplify the presentation, we use the following notations:

$$
\lambda^{k}=\lambda\left(\rho^{k}\right) \text { and } \mu^{k}=\mu\left(\rho^{k}\right) .
$$

Note that

$$
\begin{aligned}
& 0<\rho^{k} \leq C, \quad 0<\mu_{0} \leq \mu^{k}, \quad\left|\nabla \mu^{k}\right|,\left|\nabla \lambda^{k}\right| \leq \tilde{C}\left|\nabla \rho^{k}\right|, \\
& \left|\nabla^{2} \lambda^{k}\right|,\left|\nabla^{2} \mu^{k}\right| \leq \tilde{C}\left(\left|\nabla \rho^{k}\right|^{2}+\left|\nabla^{2} \rho^{k}\right|\right), \\
& \left|\nabla^{3} \lambda^{k}\right|,\left|\nabla^{3} \mu^{k}\right| \leq \tilde{C}\left(\left|\nabla \rho^{k}\right|^{3}+\left|\nabla \rho^{k}\right|\left|\nabla^{2} \rho^{k}\right|+\left|\nabla^{3} \rho^{k}\right|\right) \quad \text { on } \quad[0, T] \times \bar{\Omega},
\end{aligned}
$$

where $\tilde{C}$ depends also on $\left\|\frac{d^{j} \mu^{k}}{d \rho^{j}}\right\|_{L^{\infty}}$ and $\left\|\frac{d^{j} \lambda^{k}}{d \rho^{j}}\right\|_{L^{\infty}} j=1,2,3$.

Throughout this section, we denote some increasing continuous functions by $A\left(\Phi_{K}, t\right)$, which do not depend on $\delta$ and satisfy $\lim _{t \rightarrow 0} A\left(\Phi_{K}, t\right)=0$. Moreover we de-

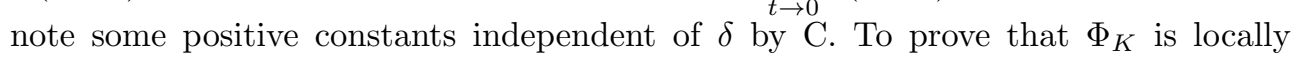
bounded, we will show

$$
\Phi_{K}(t) \leq C+A\left(\Phi_{K}, t\right)+\exp \left\{A\left(\Phi_{K}, t\right)\right\}
$$


which implies that there is a time $T_{*}>0$ such that $\Phi_{K}(t)$ is uniformly bounded to $t \in\left(0, T_{*}\right)$ and $\mathrm{K}$.

LEMMA 3.1.

$$
\begin{aligned}
& \left\|\rho^{k}\right\|_{H^{2}}+\left\|P^{k}\right\|_{H^{2}} \leq C \exp \left\{A\left(\Phi_{K}, t\right)\right\}, \\
& \int_{0}^{t}\left\|\sqrt{\rho^{k}} u_{t}^{k}\right\|_{L^{2}}^{2} d s+\left\|\nabla u^{k}(t)\right\|_{L^{2}}^{2} \leq C+A\left(\Phi_{K}, t\right)+C \exp \left\{A\left(\Phi_{K}, t\right)\right\},
\end{aligned}
$$

for all $k, 1 \leq k \leq K$.

Proof. Similar to the proof of (2.8), we can obtain (3.6). We now estimate (3.7). Multiplying (3.4) by $u_{t}^{k}$, and integrating it over $\Omega$, we obtain

$$
\begin{aligned}
& \int \rho^{k}\left|u_{t}^{k}\right|^{2} d x+\frac{d}{d t} \int\left(\mu^{k}\left|\mathcal{D} u^{k}\right|^{2}+\frac{\lambda^{k}}{2}\left(\operatorname{div} u^{k}\right)^{2}-P^{k} \operatorname{div} u^{k}\right) d x \\
= & \int\left(\left(\mu^{k}\right)^{\prime} \rho_{t}^{k}\left|\mathcal{D} u^{k}\right|^{2}+\frac{\left(\lambda^{k}\right)^{\prime}}{2} \rho_{t}^{k}\left(\operatorname{div} u^{k}\right)^{2}\right) d x \\
& -\int\left(\rho^{k} u^{k-1} \cdot \nabla u^{k} \cdot u_{t}^{k}+P_{t}^{k} \operatorname{div} u^{k}\right) d x \\
\leq & C \int\left|\rho_{t}^{k}\right|\left|\nabla u^{k}\right|^{2} d x+\int\left(\rho^{k}\left|u^{k-1}\right|^{2}\left|\nabla u^{k}\right|^{2}+\left|P_{t}^{k}\right|\left|\nabla u^{k}\right|\right) d x+\frac{1}{2} \int \rho^{k}\left|u_{t}^{k}\right|^{2} d x .
\end{aligned}
$$

Integrating (3.8), using Lemma 2.1 and (3.3), we deduce that

$$
\begin{aligned}
& \int_{0}^{t} \int \rho^{k}\left|u_{t}^{k}\right|^{2} d x d s+\int\left|\nabla u^{k}\right|^{2} d x \\
& \leq C+C \int\left|P^{k}(t)\right|^{2} d x+\int_{0}^{t} \int\left|\rho_{t}^{k} \| \nabla u^{k}\right|^{2} d x d s \\
& \quad+\int_{0}^{t} \int \rho^{k}\left|u^{k-1}\right|^{2}\left|\nabla u^{k}\right|^{2} d x d s+\int_{0}^{t} \int\left|P_{t}^{k} \| \nabla u^{k}\right| d x d s \\
& \leq C+C \int\left|P^{k}(t)\right|^{2} d x+\int_{0}^{t}\left\|\nabla \rho^{k}\right\|_{L^{6}}\left\|u^{k-1}\right\|_{L^{6}}\left\|\nabla u^{k}\right\|_{L^{3}}^{2} d s \\
& \quad+C \int_{0}^{t}\left\|\rho^{k}\right\|_{L^{\infty}}\left\|\nabla u^{k-1}\right\|_{L^{2}}\left\|\nabla u^{k}\right\|_{L^{4}}^{2} d s+C \int_{0}^{t}\left(\left\|\rho^{k}\right\|_{L^{\infty}}\left\|u^{k-1}\right\|_{L^{6}}^{2}\left\|\nabla u^{k}\right\|_{L^{3}}^{2}\right. \\
& \left.\quad+\left\|\nabla P^{k}\right\|_{L^{3}}\left\|u^{k-1}\right\|_{L^{6}}\left\|\nabla u^{k}\right\|_{L^{2}}+\left\|P^{k}\right\|_{L^{\infty}}\left\|\nabla u^{k-1}\right\|_{L^{2}}\left\|\nabla u^{k}\right\|_{L^{2}}\right) d s .
\end{aligned}
$$

Hence (3.7) follows from Sobolev's inequality and (3.9).

LEMMA 3.2.

$$
\left\|\sqrt{\rho^{k}} u_{t}^{k}\right\|_{L^{2}}^{2}+\left\|u^{k}\right\|_{H^{2}}^{2}+\int_{0}^{t}\left\|u_{t}^{k}\right\|_{H_{0}^{1}}^{2} d s \leq C+A\left(\Phi_{K}, t\right)+C \exp \left\{A\left(\Phi_{K}, t\right)\right\},
$$

for all $k, 1 \leq k \leq K$.

Proof. Differentiating (3.4) with respect to t, we get

$$
\begin{array}{r}
\rho^{k} u_{t t}^{k}+\rho_{t}^{k} u_{t}^{k}+\left(\rho^{k} u^{k-1} \cdot \nabla u^{k}\right)_{t}-\operatorname{div}\left(2 \mu \mathcal{D} u_{t}^{k}\right)-\nabla\left(\lambda^{k} \operatorname{div} u_{t}^{k}\right)+\nabla P_{t}^{k} \\
-\operatorname{div}\left(2\left(\mu^{k}\right)^{\prime} \rho_{t}^{k} \mathcal{D} u^{k}\right)-\nabla\left(\left(\lambda^{k}\right)^{\prime} \rho_{t}^{k} \operatorname{div} u^{k}\right)=0 .
\end{array}
$$


Multiplying (3.11) by $u_{t}^{k}$ and then integrating the resulting equation over $\Omega$, one gets after integration by parts

$$
\begin{gathered}
\quad \frac{1}{2} \frac{d}{d t} \int \rho^{k}\left|u_{t}^{k}\right|^{2} d x+\int\left(2 \mu^{k}\left|\mathcal{D} u_{t}^{k}\right|^{2}+\lambda^{k}\left(\operatorname{div} u_{t}^{k}\right)^{2}\right) d x \\
=\int \operatorname{div}\left(\rho^{k} u^{k-1}\right)\left(u_{t}^{k}+u^{k-1} \cdot \nabla u^{k}\right) \cdot u_{t}^{k} d x-\int \rho^{k} u_{t}^{k-1} \cdot \nabla u^{k} \cdot u_{t}^{k} d x \\
\quad-\int \mu_{t}^{k}\left(\left|\mathcal{D} u^{k}\right|^{2}\right)_{t} d x-\frac{1}{2} \int \lambda_{t}^{k}\left(\left(\operatorname{div} u^{k}\right)^{2}\right)_{t} d x+\int P_{t}^{k} \operatorname{div} u_{t}^{k} d x \\
=-\int \rho^{k} u^{k-1} \nabla\left(\left(u_{t}^{k}+u^{k-1} \cdot \nabla u^{k}\right) \cdot u_{t}^{k}\right) d x-\int \rho^{k} u_{t}^{k-1} \cdot \nabla u^{k} \cdot u_{t}^{k} d x \\
\quad-\int\left(u^{k-1} \cdot \nabla P+\gamma P \operatorname{div} u^{k-1}\right) \operatorname{div} u_{t}^{k} d x \\
\quad-\int \mu_{t}^{k}\left(\left|\mathcal{D} u^{k}\right|^{2}\right)_{t} d x-\frac{1}{2} \int \lambda_{t}^{k}\left(\left(\operatorname{div} u^{k}\right)^{2}\right)_{t} d x .
\end{gathered}
$$

Using the equation (3.3), we deduce that

$$
\begin{aligned}
& \frac{1}{2} \frac{d}{d t} \int \rho^{k}\left|u_{t}^{k}\right|^{2} d x+C \int\left|\nabla u_{t}^{k}\right|^{2} d x \\
& \leq \int\left(2 \rho^{k}\left|u^{k-1}\right|\left|u_{t}^{k}\right|\left|\nabla u_{t}^{k}\right|+\rho^{k}\left|u^{k-1}\right|\left|u_{t}^{k}\right|\left|\nabla u^{k-1}\right|\left|\nabla u^{k}\right|+\rho^{k}\left|u^{k-1}\right|^{2}\left|u_{t}^{k}\right|\left|\nabla^{2} u^{k}\right|\right. \\
& +\rho^{k}\left|u^{k-1}\right|^{2}\left|\nabla u^{k}\right|\left|\nabla u_{t}^{k}\right|+\rho^{k}\left|u_{t}^{k-1}\right|\left|\nabla u^{k}\right|\left|u_{t}^{k}\right|+\left|\nabla P^{k}\right|\left|\operatorname{div} u_{t}^{k}\right|\left|u^{k-1}\right| \\
& +\gamma P^{k}\left|\operatorname{div} u^{k-1}\right|\left|\operatorname{div} u_{t}^{k}\right| \\
& \left.+\left(2\left|\left(\mu^{k}\right)^{\prime}\right| \rho^{k}\left|\operatorname{div} u^{k-1}\right|\left|\nabla u^{k}\right|\left|\nabla u_{t}^{k}\right|+\left|\left(\lambda^{k}\right)^{\prime}\right|\left|\nabla \rho^{k}\right|\left|u^{k-1}\right|\left|\operatorname{div} u^{k}\right|\left|\operatorname{div} u_{t}^{k}\right|\right)\right) d x \\
& :=\sum_{j=1}^{8} I_{j}
\end{aligned}
$$

Using Sobolev's inequality, Hölder's inequality, (3.6), and (3.7), we get

$$
\begin{aligned}
& I_{1} \leq 2\left\|\rho^{k}\right\|_{L^{\infty}}^{\frac{1}{2}}\left\|u^{k-1}\right\|_{L^{\infty}}\left\|\sqrt{\rho^{k}} u_{t}^{k}\right\|_{L^{2}}\left\|\nabla u_{t}^{k}\right\|_{L^{2}} \leq M\left(\Phi_{K}\right), \\
& I_{2} \leq\left\|\rho^{k}\right\|_{L^{\infty}}\left\|u^{k-1}\right\|_{L^{\infty}}\left\|\nabla u^{k-1}\right\|_{L^{2}}\left\|\nabla u^{k}\right\|_{L^{3}}\left\|u_{t}^{k}\right\|_{L^{6}} \leq M\left(\Phi_{K}\right), \\
& I_{3} \leq\left\|\rho^{k}\right\|_{L^{\infty}}\left\|u^{k-1}\right\|_{L^{6}}^{2}\left\|u_{t}^{k}\right\|_{L^{6}}\left\|\nabla^{2} u^{k}\right\|_{L^{2}} \leq M\left(\Phi_{K}\right), \\
& I_{4} \leq\left\|\rho^{k}\right\|_{L^{\infty}}\left\|u^{k-1}\right\|_{L^{6}}^{2}\left\|\nabla u^{k}\right\|_{L^{6}}\left\|\nabla u_{t}^{k}\right\|_{L^{2}} \leq M\left(\Phi_{K}\right), \\
& I_{5} \leq\left\|\rho^{k}\right\|_{L^{6}}\left\|u_{t}^{k-1}\right\|_{L^{6}}\left\|\nabla u^{k}\right\|_{L^{2}}\left\|u_{t}^{k}\right\|_{L^{6}} \leq M\left(\Phi_{K}\right), \\
& I_{6} \leq\left\|\nabla P^{k}\right\|_{L^{3}}\left\|u^{k-1}\right\|_{L^{6}}\left\|\nabla u_{t}^{k}\right\|_{L^{2}} \leq M\left(\Phi_{K}\right), \\
& I_{7} \leq\left\|\gamma P\left(\rho^{k}\right)\right\|_{L^{\infty}}\left\|\nabla u^{k-1}\right\|_{L^{2}}\left\|\nabla u_{t}^{k}\right\|_{L^{2}} \leq M\left(\Phi_{K}\right), \\
& I_{8} \leq C\left(\left\|\rho^{k}\right\|_{L^{6}}\left\|\nabla u^{k-1}\right\|_{L^{6}}+\left\|\nabla \rho^{k}\right\|_{L^{6}}\left\|u^{k-1}\right\|_{L^{6}}\right)\left\|\nabla u^{k}\right\|_{L^{6}}\left\|\nabla u_{t}^{k}\right\|_{L^{2}} \leq M\left(\Phi_{K}\right),
\end{aligned}
$$

where $M=M(\cdot)$ is an increasing continuous function from $[0, \infty)$ to itself with $M(0)=$ 0 , which is independent of $\delta$.

Inserting all the estimates of $I_{i},(i=1,2, \cdots, 8)$ into (3.12) and integrating it over $[\tau, t], 0<\tau<t \leq T$, we obtain

$$
\frac{d}{d t} \int \rho^{k}\left|u_{t}^{k}\right|^{2} d x+\int\left|\nabla u_{t}^{k}\right|^{2} d x \leq M\left(\Phi_{K}\right)
$$


and

$$
\left\|\sqrt{\rho^{k}} u_{t}^{k}\right\|_{L^{2}}^{2}+\int_{\tau}^{t}\left\|\nabla u_{t}^{k}\right\|_{L^{2}}^{2} d s \leq \int \rho^{k}\left|u_{t}^{k}\right|^{2}(\tau) d x+\int_{\tau}^{t} M\left(\Phi_{K}\right) d s .
$$

Multiplying (3.4) by $u_{t}^{k}$ and integrating over $\Omega$, we have

$$
\int \rho^{k}\left|u_{t}^{k}\right|^{2} d x \leq 2 \int\left(\rho^{k}\left|u^{k-1}\right|^{2}\left|\nabla u^{k}\right|^{2}+\left(\rho^{k}\right)^{-1}\left|L u^{k}+\nabla P^{k}\right|^{2}\right) d x .
$$

Therefore, letting $\tau \rightarrow 0$ in (3.13), we conclude that

$$
\left\|\sqrt{\rho^{k}} u_{t}^{k}\right\|_{L^{2}}^{2}+\int_{0}^{t}\left\|u_{t}^{k}\right\|_{H^{1}}^{2} d s \leq \mathcal{C}\left(\rho_{0}, u_{0}\right)+A\left(\Phi_{K}, t\right)
$$

for all $k, 1 \leq k \leq K$, where

$$
\begin{aligned}
\mathcal{C}\left(\rho_{0 \delta}, u_{0 \delta}\right) & :=2 \int\left(\rho_{0 \delta}\left|u_{0 \delta}\right|^{2}\left|\nabla u_{0 \delta}\right|^{2}+\rho_{0 \delta}^{-1} \mid-\operatorname{div}\left(2 \mu\left(\rho_{0 \delta}\right) \mathcal{D} u_{0 \delta}\right)-\nabla\left(\lambda\left(\rho_{0 \delta}\right) \operatorname{div} u_{0 \delta}\right)\right. \\
& \left.+\left.\nabla P\left(\rho_{0 \delta}\right)\right|^{2}\right) d x \\
& =2 \int\left(\rho_{0 \delta}\left|u_{0 \delta}\right|^{2}\left|\nabla u_{0 \delta}\right|^{2}+\rho_{0 \delta}|g|^{2}\right) d x
\end{aligned}
$$

according to the compatibility condition (1.6) in Theorem 1.1, we may require that $\mathcal{C}\left(\rho_{0 \delta}, u_{0 \delta}\right)$ is uniformly bounded to $\delta$.

Note that for fixed $t>0,(3.4)$ is a elliptic system. According to Lemma 2.1 and Lemma 2.3, (3.4) implies

$$
\begin{aligned}
& \quad\left\|\nabla^{2} u^{k}\right\|_{L^{2}} \\
& \leq C\left(\left\|\rho^{k} u_{t}^{k}+\rho u^{k-1} \cdot \nabla u^{k}+\nabla P^{k}\right\|_{L^{2}}+\left\|2\left(\mu^{k}\right)^{\prime} \nabla \rho^{k} \cdot \mathcal{D} u^{k}-\left(\lambda^{k}\right)^{\prime} \nabla \rho^{k} \operatorname{div} u^{k}\right\|_{L^{2}}\right. \\
& \left.\quad+\left\|\nabla u^{k}\right\|_{L^{2}}\right) \\
& \leq C\left(\left\|\rho^{k}\right\|_{L^{\frac{1}{2}}}^{\frac{1}{2}}\left\|\left(\rho^{k}\right)^{\frac{1}{2}} u_{t}^{k}\right\|_{L^{2}}+\left\|\rho^{k} u^{k-1} \cdot \nabla u^{k}\right\|_{L^{2}}+\left\|\nabla P^{k}\right\|_{L^{2}}+\left\|\nabla \rho^{k}\right\|_{L^{6}}\left\|\nabla u^{k}\right\|_{L^{3}}\right. \\
& \left.\quad+\left\|\nabla u^{k}\right\|_{L^{2}}\right) \\
& \quad C C+A\left(\Phi_{K}, t\right)+C \exp \left\{A\left(\Phi_{K}, t\right)\right\}+\frac{1}{2}\left\|\nabla^{2} u^{k}\right\|_{L^{2}},
\end{aligned}
$$

where estimates (3.6), (3.7), and (3.14) are used. Hence we have

$$
\left\|\nabla^{2} u^{k}\right\|_{L^{2}} \leq C+A\left(\Phi_{K}, t\right)+C \exp \left\{A\left(\Phi_{K}, t\right)\right\} .
$$

Combing (3.14) and (3.15), we get (3.10).

LEMMa 3.3 .

$$
\begin{gathered}
\left\|\nabla u_{t}^{k}\right\|_{L^{2}}^{2}+\left\|u^{k}\right\|_{H^{3}}^{2}+\left\|\rho^{k}\right\|_{H^{3}}^{2}+\left\|P^{k}\right\|_{H^{3}}^{2} \\
+\int_{0}^{t}\left(\left\|\sqrt{\rho^{k}} u_{t t}^{k}\right\|_{L^{2}}^{2}+\left\|\nabla^{2} u_{t}^{k}\right\|_{L^{2}}^{2}+\left\|\nabla^{2} u^{k}\right\|_{H^{2}}^{2}\right) d s \\
\leq C+A\left(\Phi_{K}, t\right)+C \exp \left\{A\left(\Phi_{K}, t\right)\right\} .
\end{gathered}
$$


Proof. Multiplying (3.11) by $u_{t t}^{k}$ and then integrating the resulting equation over $\Omega$, one gets after integration by parts

$$
\begin{aligned}
\frac{d}{d t} \int\left(\mu^{k}\left|\mathcal{D} u_{t}^{k}\right|^{2}+\frac{\lambda^{k}}{2}\left(\operatorname{div} u_{t}^{k}\right)^{2}\right) d x+\int \rho^{k}\left|u_{t t}^{k}\right|^{2} d x \\
=\frac{d}{d t}\left(-\frac{1}{2} \int \rho_{t}^{k}\left|u_{t}^{k}\right|^{2}-\int \rho_{t}^{k} u^{k-1} \cdot \nabla u^{k} \cdot u_{t}^{k}+\int P_{t}^{k} \operatorname{div} u_{t}^{k} d x\right. \\
\left.\quad-\int\left(2\left(\mu^{k}\right)^{\prime} \rho_{t}^{k} \mathcal{D} u^{k} \cdot \nabla u_{t}^{k}+\left(\lambda^{k}\right)^{\prime} \rho_{t}^{k} \operatorname{div} u^{k} \operatorname{div} u_{t}^{k}\right) d x\right) \\
+\frac{1}{2} \int \rho_{t t}^{k}\left|u_{t}^{k}\right|^{2} d x+\int\left(\rho_{t}^{k} u^{k-1} \cdot \nabla u^{k}\right)_{t} \cdot u_{t}^{k} d x-\int \rho^{k} u_{t}^{k-1} \cdot \nabla u^{k} \cdot u_{t t}^{k} d x \\
\quad-\int \rho^{k} u^{k-1} \cdot \nabla u_{t}^{k} \cdot u_{t t}^{k} d x-\int P_{t t}^{k} \operatorname{div} u_{t}^{k} d x \\
+\int\left(\left(\mu^{k}\right)^{\prime} \rho_{t}^{k}\left|\mathcal{D} u_{t}^{k}\right|^{2}+\frac{\left(\lambda^{k}\right)^{\prime}}{2} \rho_{t}^{k}\left(\operatorname{div} u_{t}^{k}\right)^{2}\right) d x \\
\quad+\int\left(\left(2\left(\mu^{k}\right)^{\prime} \rho_{t}^{k} \mathcal{D} u^{k}\right)_{t} \cdot \nabla u_{t}^{k}+\left(\left(\lambda^{k}\right)^{\prime} \rho_{t}^{k} \operatorname{div} u^{k}\right)_{t} \operatorname{div} u_{t}^{k}\right) d x \\
\quad \\
:=\frac{d}{d t} J_{0}+\sum_{i=1}^{7} J_{i} .
\end{aligned}
$$

It follows from (3.6), (3.7), and (3.10) that

$$
\begin{aligned}
\left|J_{0}\right|=\mid & -\frac{1}{2} \int \rho_{t}^{k}\left|u_{t}^{k}\right|^{2}-\int \rho_{t}^{k} u^{k-1} \cdot \nabla u^{k} \cdot u_{t}^{k}+\int P_{t}^{k} \operatorname{div} u_{t}^{k} d x \\
& \quad-\int\left(2\left(\mu^{k}\right)^{\prime} \rho_{t}^{k} \mathcal{D} u^{k} \cdot \nabla u_{t}^{k}+\left(\lambda^{k}\right)^{\prime} \rho_{t}^{k} \operatorname{div} u^{k} \operatorname{div} u_{t}^{k}\right) d x \mid \\
\leq & \left.\left|\rho^{k} u^{k-1} \cdot \nabla\right| u_{t}^{k}\right|^{2}\left|d x+C \int\right| \gamma P^{k} \operatorname{div} u^{k-1}+\nabla P^{k} \cdot u^{k-1} \| \operatorname{div} u_{t}^{k} \mid d x \\
& +C \int\left|\rho^{k} \operatorname{div} u^{k-1}+\nabla \rho^{k} \cdot u^{k-1}\right|\left(\left|\nabla u^{k}\left\|\nabla u_{t}^{k}|+| u^{k-1}\right\| \nabla u^{k} \| u_{t}^{k}\right|\right) d x \\
\leq C & \left(\left\|\rho^{k}\right\|_{L^{\infty}}^{\frac{1}{2}}\left\|u^{k-1}\right\|_{L^{\infty}}\left\|\left(\rho^{k}\right)^{\frac{1}{2}} u_{t}^{k}\right\|_{L^{2}}\left\|\nabla u_{t}^{k}\right\|_{L^{2}}+\left\|P^{k}\right\|_{L^{\infty}}\left\|\nabla u^{k-1}\right\|_{L^{2}}\left\|\nabla u_{t}^{k}\right\|_{L^{2}}\right. \\
& +\left\|u^{k-1}\right\|_{L^{\infty}}\left\|\nabla P^{k}\right\|_{L^{2}}\left\|\nabla u_{t}^{k}\right\|_{L^{2}}+\left\|\rho^{k}\right\|_{L^{\infty}}\left\|\nabla u^{k-1}\right\|_{L^{6}}\left\|\nabla u^{k}\right\|_{L^{3}}\left\|\nabla u_{t}^{k}\right\|_{L^{2}} \\
& +\left\|\nabla \rho^{k}\right\|_{L^{6}}\left\|u^{k-1}\right\|_{L^{\infty}}\left\|\nabla u^{k}\right\|_{L^{3}}\left\|\nabla u_{t}^{k}\right\|_{L^{2}}+\left\|\nabla \rho^{k}\right\|_{L^{2}}\left\|u^{k-1}\right\|_{L^{\infty}}^{2}\left\|\nabla u^{k}\right\|_{L^{3}}\left\|u_{t}^{k}\right\|_{L^{6}} \\
& \left.+\left\|\rho^{k}\right\|_{L^{\infty}}\left\|\nabla u^{k-1}\right\|_{L^{3}}\left\|u^{k-1}\right\|_{L^{\infty}}\left\|\nabla u^{k}\right\|_{L^{2}}\left\|u_{t}^{k}\right\|_{L^{6}}\right) \\
\leq & A\left(\Phi_{K}, t\right)+C \exp \left\{A\left(\Phi_{K}, t\right)\right\}+\varepsilon\left\|\nabla u_{t}^{k}\right\|_{L^{2}}^{2}
\end{aligned}
$$

and

$$
\begin{aligned}
2\left|J_{1}\right| & =\left.2\left|\int \rho_{t t}^{k}\right| u_{t}^{k}\right|^{2} d x|=2| \int\left(\rho_{t}^{k} u^{k-1}+\rho^{k} u_{t}^{k-1}\right) \nabla\left(\left|u_{t}^{k}\right|^{2}\right) d x \mid \\
& \leq C\left(\left\|\rho_{t}^{k}\right\|_{L^{\infty}}\left\|u^{k-1}\right\|_{L^{3}}+\left\|\rho^{k}\right\|_{L^{\infty}}\left\|u_{t}^{k-1}\right\|_{L^{3}}\right)\left\|u_{t}^{k}\right\|_{L^{6}}\left\|\nabla u_{t}^{k}\right\|_{L^{2}} \\
& \leq M\left(\Phi_{K}\right),
\end{aligned}
$$

where we have used

$$
\left\|\rho_{t}^{k}\right\|_{L^{\infty}} \leq C\left\|\rho_{t}^{k}\right\|_{H^{2}}=C\left\|\operatorname{div}\left(\rho^{k} u^{k-1}\right)\right\|_{H^{2}} \leq C\left\|\rho^{k}\right\|_{H^{3}}\left\|u^{k-1}\right\|_{H^{3}} \leq M\left(\Phi_{K}\right) .
$$


Sobolev's inequality and Hölder's inequality give

$$
\begin{aligned}
\left|J_{2}\right| & =\left|\int\left(\rho_{t}^{k} u^{k-1} \cdot \nabla u^{k}\right)_{t} \cdot u_{t}^{k} d x\right| \\
& =\left|\int\left(\rho_{t t}^{k} u^{k-1} \cdot \nabla u^{k}+\rho_{t}^{k} u_{t}^{k-1} \cdot \nabla u^{k}+\rho_{t}^{k} u^{k-1} \cdot \nabla u_{t}^{k}\right) \cdot u_{t}^{k} d x\right| \\
& \leq\left\|\rho_{t t}^{k}\right\|_{L^{2}}\left\|u^{k-1} \cdot \nabla u^{k}\right\|_{L^{3}}\left\|u_{t}^{k}\right\|_{L^{6}}+\left\|\rho_{t}^{k}\right\|_{L^{2}}\left\|u_{t}^{k-1}\right\|_{L^{6}}\left\|u_{t}^{k}\right\|_{L^{6}}\left\|\nabla u^{k}\right\|_{L^{6}} \\
& \quad+\left\|\rho_{t}^{k}\right\|_{L^{\infty}}\left\|u^{k-1}\right\|_{L^{3}}\left\|\nabla u_{t}^{k}\right\|_{L^{2}}\left\|u_{t}^{k}\right\|_{L^{6}} \\
& \leq M\left(\Phi_{K}\right),
\end{aligned}
$$

where we have used

$$
\begin{aligned}
\left\|\rho_{t t}^{k}\right\|_{L^{2}}= & \left\|\rho_{t}^{k} \operatorname{div} u^{k-1}+\rho^{k} \operatorname{div} u_{t}^{k-1}+\nabla \rho_{t}^{k} \cdot u^{k-1}+\nabla \rho^{k} \cdot u_{t}^{k-1}\right\|_{L^{2}} \\
\leq & C\left(\left\|\rho_{t}^{k}\right\|_{L^{6}}\left\|\nabla u^{k-1}\right\|_{L^{3}}+\left\|\rho^{k}\right\|_{L^{\infty}}\left\|\nabla u_{t}^{k-1}\right\|_{L^{2}}\right. \\
& \left.\quad+\left\|\nabla \rho_{t}^{k}\right\|_{L^{2}}\left\|u^{k-1}\right\|_{L^{\infty}}+\left\|\nabla \rho^{k}\right\|_{L^{3}}\left\|u_{t}^{k-1}\right\|_{L^{6}}\right) \\
\leq & M\left(\Phi_{K}\right) .
\end{aligned}
$$

Similarly, we have

$$
\left\|P_{t t}^{k}\right\|_{L^{2}} \leq M\left(\Phi_{K}\right)
$$

Using Cauchy's inequality, we have

$$
\begin{aligned}
&\left|J_{3}\right|+\left|J_{4}\right|=\left|\int \rho^{k} u_{t}^{k-1} \cdot \nabla u^{k} \cdot u_{t t}^{k} d x\right|+\left|\int \rho^{k} u^{k-1} \cdot \nabla u_{t}^{k} \cdot u_{t t}^{k} d x\right| \\
& \leq\left\|\rho^{k}\right\|_{L^{\infty}}^{\frac{1}{2}}\left\|\left(\rho^{k}\right)^{\frac{1}{2}} u_{t t}^{k}\right\|_{L^{2}}\left(\left\|u_{t}^{k-1}\right\|_{L^{6}}\left\|\nabla u^{k}\right\|_{L^{3}}+\left\|u^{k-1}\right\|_{L^{\infty}}\left\|\nabla u_{t}^{k}\right\|_{L^{2}}\right) \\
& \leq \varepsilon\left\|\left(\rho^{k}\right)^{\frac{1}{2}} u_{t t}^{k}\right\|_{L^{2}}^{2}+M\left(\Phi_{K}\right) \\
&\left|J_{5}\right|=\left|\int P_{t t}^{k} \operatorname{div} u_{t}^{k} d x\right| \leq C\left\|P_{t t}^{k}\right\|_{L^{2}}\left\|\nabla u_{t}^{k}\right\|_{L^{2}} \leq M\left(\Phi_{K}\right) \\
&\left|J_{6}\right|=\left|\int\left(\left(\mu^{k}\right)^{\prime} \rho_{t}^{k}\left|\mathcal{D} u_{t}^{k}\right|^{2}+\frac{\left(\lambda^{k}\right)^{\prime}}{2} \rho_{t}^{k}\left(\operatorname{div} u_{t}^{k}\right)^{2}\right) d x\right| \\
& \leq C\left\|\rho_{t}^{k}\right\|_{L^{\infty}}\left\|\nabla u_{t}^{k}\right\|_{L^{2}}^{2} \leq M\left(\Phi_{K}\right)
\end{aligned}
$$

and

$$
\begin{aligned}
\left|J_{7}\right| & =\left|\int\left(\left(2 \mu^{k} \rho_{t}^{k} \mathcal{D} u^{k}\right)_{t} \cdot \nabla u_{t}^{k}+\left(\lambda^{k} \rho_{t}^{k} \operatorname{div} u^{k}\right)_{t} \operatorname{div} u_{t}^{k}\right) d x\right| \\
& \leq C \int\left(\left|\rho_{t}^{k}\right|^{2}\left|\mathcal{D} u^{k}\right|+\left|\rho_{t t}^{k}\right| \mathcal{D} u^{k}|+| \rho_{t}^{k}|| \mathcal{D} u_{t}^{k} \mid\right)\left|\nabla u_{t}^{k}\right| d x \\
& \leq C\left(\left\|\rho_{t}^{k}\right\|_{L^{\infty}}^{2}\left\|\nabla u^{k}\right\|_{L^{2}}\left\|\nabla u_{t}^{k}\right\|_{L^{2}}+\left\|\rho_{t t}^{k}\right\|_{L^{2}}\left\|\nabla u^{k-1}\right\|_{L^{\infty}}\left\|\nabla u_{t}^{k}\right\|_{L^{2}}\right. \\
& \left.\quad+\left\|\rho_{t}^{k}\right\|_{L^{\infty}}\left\|\nabla u_{t}^{k}\right\|_{L^{2}}^{2}\right) \\
& \leq M\left(\Phi_{K}\right) .
\end{aligned}
$$

Substituting (3.18)-(3.24) into (3.17), then integrating over $[0, t]$, choosing $\varepsilon>0$ suitable small, one has

$$
\left\|\nabla u_{t}^{k}\right\|_{L^{2}}^{2}+\int_{0}^{t}\left\|\sqrt{\rho^{k}} u_{t t}^{k}\right\|_{L^{2}}^{2} d s \leq C+A\left(\Phi_{K}, t\right)+C \exp \left\{A\left(\Phi_{K}, t\right)\right\} .
$$


Using (3.6), (3.7), (3.10), (3.25), and the regularity theory for elliptic systems, we obtain from (3.4) that

$$
\begin{aligned}
& \quad\left\|\nabla^{3} u^{k}\right\|_{L^{2}} \\
& \leq C\left\|\nabla\left(\rho^{k} u_{t}^{k}+\rho u^{k-1} \cdot \nabla u^{k}+\nabla P^{k}\right)\right\|_{L^{2}} \\
& \quad+C\left\|\nabla\left(2\left(\mu^{k}\right)^{\prime} \nabla \rho^{k} \cdot \mathcal{D} u^{k}+\left(\lambda^{k}\right)^{\prime} \nabla \rho^{k} \operatorname{div} u^{k}\right)\right\|_{L^{2}}+C\left\|\nabla^{2} u^{k}\right\|_{L^{2}} \\
& \leq C\left(\left\|\nabla\left(\rho^{k} u_{t}^{k}\right)\right\|_{L^{2}}+\left\|\nabla\left(\rho^{k} u^{k-1} \cdot \nabla u^{k}\right)\right\|_{L^{2}}+\left\|\nabla^{2} P^{k}\right\|_{L^{2}}\right. \\
& \quad+\left\|\left|\nabla \rho^{k}\left\|\nabla^{2} u^{k}\left|\left\|_{L^{2}}+\right\|\left(\left|\nabla \rho^{k}\right|^{2}+\left|\nabla^{2} \rho^{k}\right|\right)\right| \nabla u^{k} \mid\right\|_{L^{2}}+\left\|\nabla^{2} u^{k}\right\|_{L^{2}}\right)\right. \\
& \leq C\left(\left\|\rho^{k}\right\|_{L^{\infty}}\left\|\nabla u_{t}^{k}\right\|_{L^{2}}+\left\|\nabla \rho^{k}\right\|_{L^{3}}\left\|u_{t}^{k}\right\|_{L^{6}}+\left\|\nabla \rho^{k}\right\|_{L^{6}}\left\|u^{k-1}\right\|_{L^{\infty}}\left\|\nabla u^{k}\right\|_{L^{3}}\right. \\
& \quad+\left\|\rho^{k}\right\|_{L^{\infty}}\left\|\nabla u^{k-1}\right\|_{L^{3}}\left\|\nabla u^{k}\right\|_{L^{6}}+\left\|\rho^{k}\right\|_{L^{\infty}}\left\|\nabla u^{k-1}\right\|_{L^{\infty}}\left\|\nabla^{2} u^{k}\right\|_{L^{2}}+\left\|\nabla^{2} P^{k}\right\|_{L^{2}} \\
& \left.\quad+\left\|\nabla \rho^{k}\right\|_{L^{\infty}}\left\|\nabla^{2} u^{k}\right\|_{L^{2}}+\left\|\nabla \rho^{k}\right\|_{L^{\infty}}^{2}\left\|\nabla u^{k}\right\|_{L^{2}}+\left\|\nabla^{2} \rho^{k}\right\|_{L^{3}}\left\|\nabla u^{k}\right\|_{L^{6}}+\left\|\nabla^{2} u^{k}\right\|_{L^{2}}\right) \\
& \leq C+A\left(\Phi_{K}, t\right)+C \exp \left\{A\left(\Phi_{K}, t\right)\right\} .
\end{aligned}
$$

Hence combining (3.10) and (3.26), we get

$$
\left\|u^{k}\right\|_{H^{3}} \leq C+A\left(\Phi_{K}, t\right)+C \exp \left\{A\left(\Phi_{K}, t\right)\right\} .
$$

Using the $L^{2}$-estimate for elliptic systems, (3.6), (3.7), (3.10), (3.25)-(3.27), we find from (3.11) that

$$
\begin{gathered}
\left\|\nabla^{2} u_{t}^{k}\right\|_{L^{2}} \leq C\left\|\rho^{k} u_{t t}^{k}+\rho_{t}^{k} u_{t}^{k}+\left(\rho^{k} u^{k-1} \cdot \nabla u^{k}\right)_{t}+\nabla P_{t}^{k}\right\|_{L^{2}} \\
+\left\|\operatorname{div}\left(2\left(\mu^{k}\right)^{\prime} \rho_{t}^{k} \mathcal{D} u^{k}\right)-\nabla\left(\left(\lambda^{k}\right)^{\prime} \rho_{t}^{k} \operatorname{div} u^{k}\right)\right\|_{L^{2}} \\
+\left\|2\left(\mu^{k}\right)^{\prime} \nabla \rho^{k} \cdot \mathcal{D} u_{t}^{k}-\left(\lambda^{k}\right)^{\prime} \nabla \rho^{k} \operatorname{div} u_{t}^{k}\right\|_{L^{2}}+C\left\|\nabla u_{t}^{k}\right\|_{L^{2}} \\
\leq C\left(\left\|\rho^{k} u_{t t}^{k}\right\|_{L^{2}}+\left\|\rho_{t}^{k}\right\|_{L^{3}}\left\|u_{t}^{k}\right\|_{L^{6}}+\left\|\rho_{t}^{k}\right\|_{L^{\infty}}\left\|u^{k-1}\right\|_{L^{\infty}}\left\|\nabla u^{k}\right\|_{L^{2}}\right. \\
+\left\|\rho^{k}\right\|_{L^{\infty}}\left\|u_{t}^{k-1}\right\|_{L^{6}}\left\|\nabla u^{k}\right\|_{L^{3}}+\left\|\rho^{k}\right\|_{L^{\infty}}\left\|u^{k-1}\right\|_{L^{\infty}}\left\|\nabla u_{t}^{k}\right\|_{L^{2}} \\
+\left\|\nabla P_{t}^{k}\right\|_{L^{2}}+\left\|\nabla \rho^{k}\right\|_{L^{\infty}}\left\|\nabla u_{t}^{k}\right\|_{L^{2}}+\left\|\rho_{t}^{k}\right\|_{L^{\infty}}\left\|\nabla u^{k}\right\|_{L^{\infty}}\left\|\nabla \rho^{k}\right\|_{L^{2}} \\
\left.+\left\|\nabla u^{k}\right\|_{L^{\infty}}\left\|\nabla \rho_{t}^{k}\right\|_{L^{2}}+\left\|\rho_{t}^{k}\right\|_{L^{\infty}}\left\|\nabla^{2} u^{k}\right\|_{L^{2}}+\left\|\nabla u_{t}^{k}\right\|_{L^{2}}\right) \\
\leq C\left\|\left(\rho^{k}\right)^{\frac{1}{2}} u_{t t}^{k}\right\|_{L^{2}}+M\left(\Phi_{K}\right)+A\left(\Phi_{K}, t\right)+C \exp \left\{A\left(\Phi_{K}, t\right)\right\}
\end{gathered}
$$

(3.28) and (3.25) imply

$$
\int_{0}^{T}\left\|\nabla^{2} u_{t}^{k}\right\|_{L^{2}}^{2} d t \leq C+A\left(\Phi_{K}, t\right)+C \exp \left\{A\left(\Phi_{K}, t\right)\right\} .
$$

Using (3.27) and the regularity theory of elliptic systems, we also have

$$
\begin{aligned}
& \quad\left\|\nabla^{4} u^{k}\right\|_{L^{2}} \\
& \leq C\left\|\nabla^{2}\left(\rho^{k} u_{t}^{k}+\rho^{k} u^{k-1} \cdot \nabla u^{k}+\nabla P^{k}\right)\right\|_{L^{2}} \\
& \quad+C\left\|\nabla^{2}\left(2\left(\mu^{k}\right)^{\prime} \nabla \rho^{k} \cdot \mathcal{D} u^{k}+\left(\lambda^{k}\right)^{\prime} \nabla \rho^{k} \operatorname{div} u^{k}\right)\right\|_{L^{2}}+C\left\|\nabla^{3} u^{k}\right\|_{L^{2}} \\
& \leq C\left(\left\|\rho^{k} u_{t}^{k}\right\|_{H^{2}}+\left\|\rho^{k} u^{k-1} \cdot \nabla u^{k}\right\|_{H^{2}}+\left\|\nabla P^{k}\right\|_{H^{2}}+\left\|\nabla \rho^{k} \cdot \nabla u^{k}\right\|_{H^{2}}\right. \\
& \left.\quad+\left\|\left|\nabla \rho^{k}\right|^{3}\left|\nabla u^{k}\right|\right\|_{L^{2}}+\left\|\left|\nabla \rho^{k}\right|^{2}\left|\nabla^{2} u^{k}\right|\right\|_{L^{2}}+\left\|\nabla^{2} \rho^{k}|| \nabla \rho^{k}\right\| \nabla u^{k} \mid\left\|_{L^{2}}+\right\| \nabla^{3} u^{k} \|_{L^{2}}\right) \\
& \leq M\left(\Phi_{K}\right)+C \Phi_{k}\left\|\nabla^{2} u_{t}^{k}\right\|_{L^{2}}+A\left(\Phi_{K}, t\right)+C \exp \left\{A\left(\Phi_{K}, t\right)\right\} .
\end{aligned}
$$

Using (3.25), (3.27), (3.29), and (3.30), we obtain

$$
\int_{0}^{t}\left\|\nabla^{4} u^{k}\right\|_{L^{2}}^{2} d s \leq A\left(\Phi_{K}, t\right)+C \exp \left\{A\left(\Phi_{K}, t\right)\right\} .
$$


Similarly to the proof of (2.8), using (3.31), one can obtain

$$
\begin{aligned}
\left\|\rho^{k}\right\|_{H^{3}} & \leq\left\|\rho_{0}\right\|_{H^{3}} \exp \left(C \int_{0}^{t}\left\|\nabla u^{k-1}\right\|_{H^{3}} d s\right) \leq C \exp \left(C t+\int_{0}^{t}\left\|u^{k-1}\right\|_{H^{4}}^{2} d s\right) \\
& \leq C \exp \left\{A\left(\Phi_{K}, t\right)\right\} .
\end{aligned}
$$

Similarly, using (2.8), we can prove

$$
\left\|P^{k}\right\|_{H^{3}} \leq\left\|P\left(\rho_{0}\right)\right\|_{H^{3}} \exp \left(C \int_{0}^{t}\left\|\nabla u^{k-1}\right\|_{H^{3}} d s\right) \leq C \exp \left\{A\left(\Phi_{K}, t\right)\right\} .
$$

The proof of Lemma 3.3 is completed.

From lemmas 3.1-3.3, we conclude that

$$
\Phi_{K}(t) \leq C+A\left(\Phi_{K}, t\right)+C \exp \left\{A\left(\Phi_{K}, t\right)\right\},
$$

for some constant $\mathrm{C}$ independent of $k$ and $\delta$. Because $\lim _{t \rightarrow 0} A\left(\Phi_{K}, t\right)=0$, there exists a time $T_{*}>0$ and a constant $\mathrm{C}$ depending on $\lambda, \mu,\|g\|_{H_{0}^{1}}$, and $\left\|\left(\rho_{0}, u_{0}\right)\right\|_{H^{3}}$ such that

$$
\Phi_{K}(t) \leq C, 0 \leq t<T_{*},
$$

for all positive integers K. Hence from lemmas 3.1-3.3, we get

$$
\begin{aligned}
\sup _{0 \leq t \leq T_{*}}\left(\left\|\rho^{k}\right\|_{H^{3}}^{2}+\left\|P^{k}\right\|_{H^{3}}^{2}+\left\|u^{k}\right\|_{H^{3}}^{2}+\left\|\sqrt{\rho^{k}} u_{t}^{k}\right\|_{L^{2}}^{2}+\left\|u_{t}^{k}\right\|_{H^{1}}^{2}\right) \\
+\int_{0}^{T_{*}}\left(\left\|u^{k}\right\|_{H^{4}}^{2}+\left\|u_{t}^{k}\right\|_{H^{2}}^{2}+\left\|\sqrt{\rho^{k}} u_{t t}^{k}\right\|_{L^{2}}^{2}\right) d t \leq C
\end{aligned}
$$

for all $k \geq 1$. Moreover, (3.33) and (3.1) 1 imply

$$
\begin{aligned}
\sup _{0 \leq t \leq T_{*}}\left(\left\|\rho_{t}^{k}\right\|_{H^{2}}^{2}\right. & \left.+\left\|P_{t}^{k}\right\|_{H^{2}}^{2}+\left\|\rho_{t t}^{k}\right\|_{L^{2}}^{2}+\left\|P_{t t}^{k}\right\|_{L^{2}}^{2}\right) \\
& +\int_{0}^{T_{*}}\left(\left\|\nabla \rho_{t t}^{k}\right\|_{L^{2}}^{2}+\left\|\nabla P_{t t}^{k}\right\|_{L^{2}}^{2}\right) d t \leq C .
\end{aligned}
$$

Then (3.32)-(3.35) imply that there is a convergent subsequence of $\left(\rho^{k}, u^{k}\right)$ such that the limit function is a strong solution of (1.1)-(1.2), which possesses the following regularity:

$$
\left\{\begin{array}{l}
\rho \in C\left(\left[0, T_{*}\right] ; H^{3}\right), \quad \rho_{t} \in C\left(\left[0, T_{*}\right] ; H^{2}\right), \\
\rho_{t t} \in L^{\infty}\left(0, T_{*} ; L^{2}\right) \cap L^{2}\left(0, T_{*} ; H^{1}\right), \\
u \in C\left(\left[0, T_{*}\right] ; H_{0}^{1} \cap H^{3}\right) \cap L^{2}\left(0, T_{*} ; H^{4}\right), \sqrt{\rho} u_{t} \in L^{\infty}\left(0, T_{*} ; L^{2}\right), \\
u_{t} \in L^{\infty}\left(0, T_{*} ; H_{0}^{1}\right) \cap L^{2}\left(0, T_{*} ; H^{2}\right), \quad \text { and } \quad \sqrt{\rho} u_{t t} \in L^{2}\left(0, T_{*} ; L^{2}\right) .
\end{array}\right.
$$

We now prove that the above strong solution is also a classical solution of (1.1)(1.2).

LEMMA 3.4. The strong solutions of (1.1)-(1.2) have the following regularity:

$$
t \sqrt{\rho} u_{t t t} \in L^{2}\left(0, T_{*} ; L^{2}\right), \quad t u_{t t} \in L^{\infty}\left(0, T_{*} ; H^{1}\right) .
$$


Proof. The following calculations are formal, and $(\rho, u)$ is required to be more regular. They can be made rigorous by a Steklov averaging process. Differentiating $(1.1)_{2}$ twice with respect to $t$, we have

$$
\begin{aligned}
\rho u_{t t t}-\operatorname{div}\left(2 \mu \mathcal{D} u_{t t}\right)-\nabla\left(\lambda \operatorname{div} u_{t t}\right)= & -\nabla P_{t t}-\rho(u \cdot \nabla u)_{t t}-2 \rho_{t}\left(u \cdot \nabla u+u_{t}\right)_{t} \\
& -\rho_{t t}\left(u \cdot \nabla u+u_{t}\right)+\operatorname{div}\left(2 \mu_{t t} \mathcal{D} u\right)+\nabla\left(\lambda_{t t} \operatorname{div} u\right) \\
& +\operatorname{div}\left(4 \mu_{t} \mathcal{D} u_{t}\right)+\nabla\left(2 \lambda_{t} \operatorname{div} u_{t}\right) .
\end{aligned}
$$

Multiplying (3.38) by $u_{t t}$ and integrating over $\Omega$, one gets after integration by parts that

$$
\begin{aligned}
& \frac{1}{2} \frac{d}{d t} \int \rho\left|u_{t t}\right|^{2} d x+\int\left(2 \mu\left|\mathcal{D} u_{t t}\right|^{2}+\lambda\left(\operatorname{div} u_{t t}\right)^{2}\right) d x \\
=\int P_{t t} & \operatorname{div} u_{t t} d x-\int \rho(u \cdot \nabla u)_{t t} \cdot u_{t t} d x-2 \int \rho_{t}(u \cdot \nabla u)_{t} \cdot u_{t t} d x \\
& -\frac{3}{2} \int \rho_{t}\left|u_{t t}\right|^{2} d x-\int \rho_{t t} u \cdot \nabla u \cdot u_{t t} d x-\int \rho_{t t} u_{t} \cdot u_{t t} d x \\
& -\int\left(2 \mu_{t t} \mathcal{D} u \cdot \nabla u_{t t}+\lambda_{t t} \operatorname{div} u \operatorname{div} u_{t t}\right) d x \\
& -\int\left(4 \mu_{t} \mathcal{D} u_{t} \cdot \nabla u_{t t}+2 \lambda_{t} \operatorname{div} u_{t} \operatorname{div} u_{t t}\right) d x \\
:= & \sum_{i=1}^{8} M_{i} .
\end{aligned}
$$

We can estimate each term of the right hand side of (3.39) as follows:

$$
\begin{gathered}
M_{1}=\int P_{t t} \operatorname{div} u_{t t} d x \leq C(\varepsilon)\left\|P_{t t}\right\|_{L^{2}}^{2}+\varepsilon\left\|\nabla u_{t t}\right\|_{L^{2}}^{2} \leq \varepsilon\left\|\nabla u_{t t}\right\|_{L^{2}}^{2}+C(\varepsilon), \\
M_{2}=-\int \rho(u \cdot \nabla u)_{t t} \cdot u_{t t} d x \\
\leq C\|\rho\|_{L^{\infty}}^{\frac{1}{2}}\left(\left\|u_{t t}\right\|_{L^{6}}\|\nabla u\|_{L^{3}}+\left\|u_{t}\right\|_{L^{6}}\left\|\nabla u_{t}\right\|_{L^{3}}+\|u\|_{L^{\infty}}\left\|\nabla u_{t t}\right\|_{L^{2}}\right)\left\|\sqrt{\rho} u_{t t}\right\|_{L^{2}} \\
\leq \varepsilon\left\|\nabla u_{t t}\right\|_{L^{2}}^{2}+C(\varepsilon)\left\|\sqrt{\rho} u_{t t}\right\|_{L^{2}}^{2}+C\left\|\nabla^{2} u_{t}\right\|_{L^{2}}^{2}, \\
M_{3}=-2 \int \rho_{t}(u \cdot \nabla u)_{t} \cdot u_{t t} d x \leq C\left\|\rho_{t}\right\|_{L^{3}}\left(\left\|u_{t}\right\|_{L^{6}}\|\nabla u\|_{L^{3}}+\|u\|_{L^{\infty}}\left\|\nabla u_{t}\right\|_{L^{2}}\right)\left\|u_{t t}\right\|_{L^{6}} \\
\leq \varepsilon\left\|\nabla u_{t t}\right\|_{L^{2}}^{2}+C(\varepsilon), \\
M_{4}=-\frac{3}{2} \int \rho_{t}\left|u_{t t}\right|^{2} d x=\frac{3}{2} \int \operatorname{div}(\rho u)\left|u_{t t}\right|^{2} d x \leq 3 \int \rho\left|u\left\|u_{t t}\right\| \nabla u_{t t}\right| d x \\
\leq C\|\rho\|_{L^{\infty}}^{\frac{1}{2}}\|u\|_{L^{\infty}}\left\|\nabla u_{t t}\right\|_{L^{2}}\left\|\sqrt{\rho} u_{t t}\right\|_{L^{2}} \leq \varepsilon\left\|\nabla u_{t t}\right\|_{L^{2}}^{2}+C(\varepsilon)\left\|\sqrt{\rho} u_{t t}\right\|_{L^{2}}^{2}, \\
M_{5}=-\int \rho_{t t} u \cdot \nabla u \cdot u_{t t} d x \leq C\left\|\rho_{t t}\right\|_{L^{2}}\|u\|_{L^{\infty}}\|\nabla u\|_{L^{3}}\left\|u_{t t}\right\|_{L^{6}} \leq \varepsilon\left\|\nabla u_{t t}\right\|_{L^{2}}^{2}+C(\varepsilon),
\end{gathered}
$$




$$
\begin{gathered}
M_{6}=-\int \rho_{t t} u_{t} \cdot u_{t t} d x \leq C\left\|\rho_{t t}\right\|_{L^{2}}\left\|u_{t}\right\|_{L^{3}}\left\|u_{t t}\right\|_{L^{6}} \leq \varepsilon\left\|\nabla u_{t t}\right\|_{L^{2}}^{2}+C(\varepsilon), \\
M_{7}=-\int\left(2 \mu_{t t} \mathcal{D} u \cdot \nabla u_{t t}+\lambda_{t t} \operatorname{div} u \operatorname{div} u_{t t}\right) d x \leq C \int\left(\left|\rho_{t t}\right|+\left|\rho_{t}\right|^{2}\right)\left|\nabla u \| \nabla u_{t t}\right| d x \\
\leq C\left(\left\|\rho_{t t}\right\|_{L^{2}}+\left\|\rho_{t}\right\|_{L^{4}}^{2}\right)\|\nabla u\|_{L^{\infty}}\left\|\nabla u_{t t}\right\|_{L^{2}} \leq \varepsilon\left\|\nabla u_{t t}\right\|_{L^{2}}^{2}+C(\varepsilon), \\
M_{8}=-\int\left(4 \mu_{t} \mathcal{D} u_{t} \cdot \nabla u_{t t}+2 \lambda_{t} \operatorname{div} u_{t} \operatorname{div} u_{t t}\right) d x \leq C \int\left|\rho_{t}\left\|\nabla u_{t}\right\| \nabla u_{t t}\right| d x \\
\leq C\left\|\rho_{t}\right\|_{L^{\infty}}\left\|\nabla u_{t}\right\|_{L^{2}}\left\|\nabla u_{t t}\right\|_{L^{2}} \leq \varepsilon\left\|\nabla u_{t t}\right\|_{L^{2}}^{2}+C(\varepsilon) .
\end{gathered}
$$

Substituting all estimates of $M_{i},(i=1,2, \cdots, 8)$ into (3.39) and choosing $\varepsilon>0$ small enough, we have

$$
\frac{d}{d t} \int \rho\left|u_{t t}\right|^{2} d x+\left\|\nabla u_{t t}\right\|_{L^{2}}^{2} \leq C\left(1+\left\|\nabla^{2} u_{t}\right\|_{L^{2}}^{2}+\left\|\sqrt{\rho} u_{t t}\right\|_{L^{2}}^{2}\right) .
$$

Multiplying (3.40) by $t$, integrating over $\left(\tau, T_{*}\right)$, and using Gronwall's inequality, we deduce that

$$
t\left\|\sqrt{\rho} u_{t t}(t)\right\|_{L^{2}}^{2}+\int_{\tau}^{T_{*}} s\left\|\nabla u_{t t}(s)\right\|_{L^{2}}^{2} d s \leq C\left(1+\tau\left\|\sqrt{\rho} u_{t t}(\tau)\right\|_{L^{2}}^{2}\right) .
$$

Because $\left\|\sqrt{\rho} u_{t t}\right\|_{L^{2}}^{2} \in L^{2}\left(0, T_{*}\right)$, it follows that there is a sequence $\left\{\tau_{k}>0\right\}$ such that

$$
\tau_{k} \rightarrow 0 \text { and } \tau_{k}\left\|\sqrt{\rho} u_{t t}\left(\tau_{k}\right)\right\|_{L^{2}}^{2} \rightarrow 0 \text { as } k \rightarrow \infty .
$$

Therefore, letting $\tau=\tau_{k} \rightarrow 0$ in (3.41), we conclude that

$$
t\left\|\sqrt{\rho} u_{t t}(t)\right\|_{L^{2}}^{2}+\int_{\tau}^{T_{*}} s\left\|\nabla u_{t t}(s)\right\|_{L^{2}}^{2} d s \leq C .
$$

Multiplying (3.38) by $u_{t t t}$ and integrating over $\Omega$, we have

$$
\begin{aligned}
& \int \rho\left|u_{t t t}\right|^{2} d x+\frac{1}{2} \frac{d}{d t} \int\left(2 \mu\left|\mathcal{D} u_{t t}\right|^{2}+\lambda\left(\operatorname{div} u_{t t}\right)^{2}\right) d x \\
& =\int P_{t t} \operatorname{div} u_{t t t} d x-\int \rho(u \cdot \nabla u)_{t t} \cdot u_{t t t} d x-2 \int \rho_{t}\left(u \cdot \nabla u+u_{t}\right)_{t} \cdot u_{t t t} d x \\
& \quad-\int \rho_{t t}(u \cdot \nabla u) \cdot u_{t t t} d x-\int \rho_{t t} u_{t} \cdot u_{t t t} d x \\
& \quad-\int\left(2 \mu_{t t} \mathcal{D} u \cdot \nabla u_{t t t}+\lambda_{t t} \operatorname{div} u \operatorname{div} u_{t t t}\right) d x \\
& \quad-\int\left(4 \mu_{t} \mathcal{D} u_{t} \cdot \nabla u_{t t t}+2 \lambda_{t} \operatorname{div} u_{t} \operatorname{div} u_{t t t}\right) d x \\
& :=\sum_{i=1}^{7} N_{i} .
\end{aligned}
$$

We can estimate the terms of the right side in (3.43) as follows:

$$
\begin{aligned}
N_{1} & =\int P_{t t} \operatorname{div} u_{t t t} d x=\frac{d}{d t} \int P_{t t} \operatorname{div} u_{t t} d x-\int P_{t t t} \operatorname{div} u_{t t} d x \\
& \leq \frac{d}{d t} \int P_{t t} \operatorname{div} u_{t t} d x+C\left(\left\|P_{t t t}\right\|_{L^{2}}^{2}+\left\|\nabla u_{t t}\right\|_{L^{2}}^{2}\right) \\
& \leq \frac{d}{d t} \int P_{t t} \operatorname{div} u_{t t} d x+C\left(1+\left\|\nabla \rho_{t t}\right\|_{L^{2}}^{2}+\left\|\nabla u_{t t}\right\|_{L^{2}}^{2}\right),
\end{aligned}
$$


where we used the fact that

$$
\begin{aligned}
\left\|\rho_{t t t}\right\|_{L^{2}}= & \left\|\rho_{t t} \operatorname{div} u+2 \rho_{t} \operatorname{div} u_{t}+\rho \operatorname{div} u_{t t}+\nabla \rho_{t t} \cdot u+2 \nabla \rho_{t} \cdot u_{t}+\nabla \rho \cdot u_{t t}\right\|_{L^{2}} \\
\leq & C\left(\left\|\rho_{t t}\right\|_{L^{2}}\|\nabla u\|_{L^{\infty}}+\left\|\rho_{t}\right\|_{L^{\infty}}\left\|\nabla u_{t}\right\|_{L^{2}}+\|\rho\|_{L^{\infty}}\left\|\nabla u_{t t}\right\|_{L^{2}}\right. \\
& \left.\quad+\left\|\nabla \rho_{t t}\right\|_{L^{2}}\|u\|_{L^{\infty}}+\left\|\nabla \rho_{t}\right\|_{L^{6}}\left\|u_{t}\right\|_{L^{3}}+\|\nabla \rho\|_{L^{3}}\left\|u_{t t}\right\|_{L^{6}}\right) \\
\leq & C\left(1+\left\|\nabla \rho_{t t}\right\|_{L^{2}}+\left\|\nabla u_{t t}\right\|_{L^{2}}\right)
\end{aligned}
$$

and notice that $P=A \rho^{\gamma}$ satisfies

$$
P_{t}+u \cdot \nabla P+\gamma P \operatorname{div} u=0 .
$$

Adapting the above proof, we can prove

$$
\left\|P_{t t t}\right\|_{L^{2}} \leq C\left(1+\left\|\nabla \rho_{t t}\right\|_{L^{2}}+\left\|\nabla u_{t t}\right\|_{L^{2}}\right) .
$$

$$
\begin{aligned}
& N_{2}=-\int \rho(u \cdot \nabla u)_{t t} \cdot u_{t t t} d x \\
& \leq C\|\rho\|_{L^{\infty}}^{\frac{1}{2}}\left(\left\|u_{t t}\right\|_{L^{6}}\|\nabla u\|_{L^{3}}+\left\|u_{t}\right\|_{L^{6}}\left\|\nabla u_{t}\right\|_{L^{3}}+\|u\|_{L^{\infty}}\left\|\nabla u_{t t}\right\|_{L^{2}}\right)\left\|\sqrt{\rho} u_{t t t}\right\|_{L^{2}} \\
& \leq \varepsilon\left\|\sqrt{\rho} u_{t t t}\right\|_{L^{2}}^{2}+C(\varepsilon)\left(\left\|\nabla u_{t t}\right\|_{L^{2}}^{2}\|\nabla u\|_{L^{3}}^{2}+\left\|\nabla u_{t t}\right\|_{L^{2}}^{2}\|u\|_{H^{2}}^{2}+\left\|\nabla u_{t}\right\|_{L^{2}}^{2}\left\|\nabla u_{t}\right\|_{L^{3}}^{2}\right) \\
& \leq \varepsilon\left\|\sqrt{\rho} u_{t t t}\right\|_{L^{2}}^{2}+C(\varepsilon)\left(1+\left\|\nabla u_{t t}\right\|_{L^{2}}^{2}+\left\|\nabla^{2} u_{t}\right\|_{L^{2}}^{2}\right) \text {, } \\
& N_{3}=-2 \int \rho_{t}\left(u \cdot \nabla u+u_{t}\right)_{t} \cdot u_{t t t} d x \\
& =-\frac{d}{d t} \int\left(2 \rho_{t}(u \cdot \nabla u)_{t} \cdot u_{t t}+\rho_{t}\left|u_{t t}\right|^{2}\right) d x+2 \int \rho_{t t}(u \cdot \nabla u)_{t} \cdot u_{t t} d x \\
& +2 \int \rho_{t}(u \cdot \nabla u)_{t t} \cdot u_{t t} d x+\int \rho_{t t}\left|u_{t t}\right|^{2} d x \\
& \leq-\frac{d}{d t} \int\left(2 \rho_{t}(u \cdot \nabla u)_{t} \cdot u_{t t}+\rho_{t}\left|u_{t t}\right|^{2}\right) d x \\
& +C\left\|\rho_{t t}\right\|_{L^{2}}\left\|u_{t t}\right\|_{L^{6}}\left(\|u\|_{L^{\infty}}\left\|\nabla u_{t}\right\|_{L^{3}}+\left\|u_{t}\right\|_{L^{6}}\|\nabla u\|_{L^{6}}\right) \\
& +C\left\|\rho_{t}\right\|_{L^{3}}\left\|u_{t t}\right\|_{L^{6}}\left(\|u\|_{L^{\infty}}\left\|\nabla u_{t t}\right\|_{L^{2}}+2\left\|u_{t}\right\|_{L^{6}}\left\|\nabla u_{t}\right\|_{L^{3}}+\left\|u_{t t}\right\|_{L^{6}}\|\nabla u\|_{L^{3}}\right) \\
& +C\left\|\rho_{t t}\right\|_{L^{2}}\left\|u_{t t}\right\|_{L^{4}}^{2} \\
& \leq-\frac{d}{d t} \int\left(2 \rho_{t}(u \cdot \nabla u)_{t} \cdot u_{t t}+\rho_{t}\left|u_{t t}\right|^{2}\right) d x+C\left(1+\left\|\nabla u_{t t}\right\|_{L^{2}}^{2}+\left\|\nabla^{2} u_{t}\right\|_{L^{2}}^{2}\right) \text {, } \\
& N_{4}=-\int \rho_{t t}(u \cdot \nabla u) \cdot u_{t t t} d x \\
& =-\frac{d}{d t} \int \rho_{t t}(u \cdot \nabla u) \cdot u_{t t} d x+\int \rho_{t t t}(u \cdot \nabla u) \cdot u_{t t} d x+\int \rho_{t t}(u \cdot \nabla u)_{t} \cdot u_{t t} d x .
\end{aligned}
$$

Note that

$$
\begin{aligned}
\int \rho_{t t t}(u \cdot \nabla u) \cdot u_{t t} d x & \leq\left\|\rho_{t t t}\right\|_{L^{2}}\|\nabla u\|_{L^{3}}\|u\|_{L^{\infty}}\left\|u_{t t}\right\|_{L^{6}} \\
& \leq C\left(1+\left\|\nabla u_{t t}\right\|_{L^{2}}^{2}+\left\|\nabla \rho_{t t}\right\|_{L^{2}}^{2}\right)
\end{aligned}
$$


and

$$
\begin{aligned}
\int \rho_{t t}(u \cdot \nabla u)_{t} \cdot u_{t t} d x & =\int\left(\rho_{t t}\left(u_{t} \cdot \nabla u\right)+\rho_{t t}\left(u \cdot \nabla u_{t}\right)\right) \cdot u_{t t} d x \\
& \leq\left(\left\|\rho_{t t}\right\|_{L^{2}}\left\|u_{t}\right\|_{L^{6}}\|\nabla u\|_{L^{6}}+\left\|\rho_{t t}\right\|_{L^{3}}\|u\|_{L^{\infty}}\left\|\nabla u_{t}\right\|_{L^{2}}\right)\left\|u_{t t}\right\|_{L^{6}} \\
& \leq C\left(1+\left\|\nabla u_{t t}\right\|_{L^{2}}^{2}+\left\|\nabla \rho_{t t}\right\|_{L^{2}}^{2}\right) .
\end{aligned}
$$

We have

$$
\begin{gathered}
N_{4} \leq-\frac{d}{d t} \int \rho_{t t}(u \cdot \nabla u) \cdot u_{t t} d x+C\left(1+\left\|\nabla u_{t t}\right\|_{L^{2}}^{2}+\left\|\nabla \rho_{t t}\right\|_{L^{2}}^{2}\right), \\
N_{5}=-\int \rho_{t t} u_{t} \cdot u_{t t t} d x \\
=-\frac{d}{d t} \int \rho_{t t} u_{t} \cdot u_{t t} d x+\int\left(\rho_{t t t} u_{t}+\rho_{t t} u_{t t}\right) \cdot u_{t t} d x \\
\leq-\frac{d}{d t} \int \rho_{t t} u_{t} \cdot u_{t t} d x+C\left(\left\|\rho_{t t t}\right\|_{L^{2}}\left\|u_{t}\right\|_{L^{3}}\left\|u_{t t}\right\|_{L^{6}}+\left\|\rho_{t t}\right\|_{L^{2}}\left\|u_{t t}\right\|_{L^{4}}^{2}\right) \\
\leq-\frac{d}{d t} \int \rho_{t t} u_{t} \cdot u_{t t} d x+C\left(1+\left\|\nabla u_{t t}\right\|_{L^{2}}^{2}+\left\|\nabla \rho_{t t}\right\|_{L^{2}}^{2}\right), \\
N_{6}=-\int\left(2 \mu_{t t} \mathcal{D} u \cdot \nabla u_{t t t}+\lambda_{t t} \operatorname{div} u \operatorname{div} u_{t t t}\right) d x \\
=-\frac{d}{d t} \int\left(2 \mu_{t t} \mathcal{D} u \cdot \nabla u_{t t}+\lambda_{t t} \operatorname{div} u \operatorname{div} u_{t t}\right) d x \\
+\int\left(\left(2 \mu_{t t} \mathcal{D} u\right)_{t} \cdot \nabla u_{t t}+\left(\lambda_{t t} \operatorname{div} u\right)_{t} \operatorname{div} u_{t t t}\right) d x .
\end{gathered}
$$

Note that

$$
\begin{aligned}
\int\left(\mu_{t t} \mathcal{D} u\right)_{t} \cdot \nabla u_{t t} d x= & \int\left(\mu_{t t t} \mathcal{D} u+\mu_{t t} \mathcal{D} u_{t}\right) \cdot \nabla u_{t t} d x \\
= & \int\left(\left(\mu^{\prime} \rho_{t t t}+3 \mu^{\prime \prime} \rho_{t} \rho_{t t}+\mu^{\prime \prime \prime} \rho_{t}^{3}\right) \mathcal{D} u+\left(\mu^{\prime} \rho_{t t}+\mu^{\prime \prime} \rho_{t}^{2}\right) \mathcal{D} u_{t}\right) \cdot \nabla u_{t t} d x \\
\leq & C\left(\left\|\rho_{t t t}\right\|_{L^{2}}+\left\|\rho_{t}\right\|_{L^{\infty}}\left\|\rho_{t t}\right\|_{L^{2}}+\left\|\rho_{t}\right\|_{L^{6}}^{3}\right)\|\nabla u\|_{L^{\infty}}\left\|\nabla u_{t t}\right\|_{L^{2}} \\
& \quad+C\left(\left\|\rho_{t t}\right\|_{L^{6}}\left\|\nabla u_{t}\right\|_{L^{3}}+\left\|\rho_{t}\right\|_{L^{\infty}}^{2}\left\|\nabla u_{t}\right\|_{L^{2}}\right)\left\|\nabla u_{t t}\right\|_{L^{2}} \\
\leq & C\left(1+\left\|\nabla \rho_{t t}\right\|_{L^{2}}^{2}+\left\|\nabla u_{t t}\right\|_{L^{2}}^{2}+\left\|\nabla^{2} u_{t}\right\|_{L^{2}}^{2}\left\|\nabla u_{t t}\right\|_{L^{2}}^{2}\right) .
\end{aligned}
$$

Similarly, we have

$$
\int\left(\lambda_{t t} \operatorname{div} u\right)_{t} \operatorname{div} u_{t t t} d x \leq C\left(1+\left\|\nabla \rho_{t t}\right\|_{L^{2}}^{2}+\left\|\nabla u_{t t}\right\|_{L^{2}}^{2}+\left\|\nabla^{2} u_{t}\right\|_{L^{2}}^{2}\left\|\nabla u_{t t}\right\|_{L^{2}}^{2}\right) .
$$

Then, we have

$$
\begin{aligned}
N_{6} \leq-\frac{d}{d t} \int & \left(2 \mu_{t t} \mathcal{D} u \cdot \nabla u_{t t}+\lambda_{t t} \operatorname{div} u \operatorname{div} u_{t t}\right) d x \\
& +C\left(1+\left\|\nabla \rho_{t t}\right\|_{L^{2}}^{2}+\left\|\nabla u_{t t}\right\|_{L^{2}}^{2}+\left\|\nabla^{2} u_{t}\right\|_{L^{2}}^{2}\left\|\nabla u_{t t}\right\|_{L^{2}}^{2}\right),
\end{aligned}
$$




$$
\begin{aligned}
& N_{7}=-\int\left(4 \mu_{t} \mathcal{D} u_{t} \cdot \nabla u_{t t t}+2 \lambda_{t} \operatorname{div} u_{t} \operatorname{div} u_{t t t}\right) d x \\
&=-\frac{d}{d t} \int\left(4 \mu_{t} \mathcal{D} u_{t} \cdot \nabla u_{t t}+2 \lambda_{t} \operatorname{div} u_{t} \operatorname{div} u_{t t}\right) d x \\
& \quad+\int\left(\left(4 \mu_{t} \mathcal{D} u_{t}\right)_{t} \cdot \nabla u_{t t}+2\left(\lambda_{t} \operatorname{div} u_{t}\right)_{t} \operatorname{div} u_{t t}\right) d x
\end{aligned}
$$

Note that

$$
\begin{aligned}
& \int\left(\mu_{t} \mathcal{D} u_{t}\right)_{t} \cdot \nabla u_{t t} d x \\
= & \int\left(\mu_{t t} \mathcal{D} u+\mu_{t} \mathcal{D} u_{t}\right) \cdot \nabla u_{t t} d x \\
= & \int\left(\left(\mu^{\prime} \rho_{t t}+\mu^{\prime \prime} \rho_{t}^{2}\right) \mathcal{D} u+\mu^{\prime} \rho_{t} \mathcal{D} u_{t}\right) \cdot \nabla u_{t t} d x \\
\leq & C\left(\left\|\rho_{t t}\right\|_{L^{2}}+\left\|\rho_{t}\right\|_{L^{4}}^{2}\right)\|\nabla u\|_{L^{\infty}}\left\|\nabla u_{t t}\right\|_{L^{2}}+C\left\|\rho_{t}\right\|_{L^{\infty}}\left\|\nabla u_{t}\right\|_{L^{2}}\left\|\nabla u_{t t}\right\|_{L^{2}} \\
\leq & C\left(1+\left\|\nabla u_{t t}\right\|_{L^{2}}^{2}\right) .
\end{aligned}
$$

Similarly, we have

$$
\int\left(\lambda_{t} \operatorname{div} u_{t}\right)_{t} \operatorname{div} u_{t t} d x \leq C\left(1+\left\|\nabla u_{t t}\right\|_{L^{2}}^{2}\right)
$$

We have

$$
N_{7} \leq-\frac{d}{d t} \int\left(4 \mu_{t} \mathcal{D} u_{t} \cdot \nabla u_{t t}+2 \lambda_{t} \operatorname{div} u_{t} \operatorname{div} u_{t t}\right) d x+C\left(1+\left\|\nabla u_{t t}\right\|_{L^{2}}^{2}\right) .
$$

Substituting the above estimates of $N_{i},(i=1,2, \cdots, 7)$ into $(3.43)$ and choosing $\varepsilon>0$ suitably small, we have

$$
\begin{aligned}
& \int \rho\left|u_{t t t}\right|^{2} d x+\frac{d}{d t} \int\left(2 \mu\left|\mathcal{D} u_{t t}\right|^{2}+\lambda\left(\operatorname{div} u_{t t}\right)^{2}\right) d x \\
\leq & \frac{d}{d t} f(t)+C\left(1+\left\|\nabla \rho_{t t}\right\|_{L^{2}}^{2}+\left\|\nabla^{2} u_{t}\right\|_{L^{2}}+\left\|\nabla u_{t t}\right\|_{L^{2}}^{2}+\left\|\nabla^{2} u_{t}\right\|_{L^{2}}^{2}\left\|\nabla u_{t t}\right\|_{L^{2}}^{2}\right)
\end{aligned}
$$

where

$$
\begin{aligned}
f(t)= & \int\left(P_{t t} \operatorname{div} u_{t t}-2 \rho_{t}(u \cdot \nabla u)_{t} \cdot u_{t t}-\rho_{t}\left|u_{t t}\right|^{2}-\rho_{t t}(u \cdot \nabla u) \cdot u_{t t}-\rho_{t t} u_{t} \cdot u_{t t}\right) d x \\
& -\int\left(2 \mu_{t t} \mathcal{D} u \cdot \nabla u_{t t}+\lambda_{t t} \operatorname{div} u \operatorname{div} u_{t t}\right) d x-\int\left(4 \mu_{t} \mathcal{D} u_{t} \cdot \nabla u_{t t}+2 \lambda_{t} \operatorname{div} u_{t} \operatorname{div} u_{t t}\right) d x
\end{aligned}
$$

Multiplying (3.44) by $t^{2}$, integrating over $\left(\tau, T_{*}\right)$, and using Gronwall's inequality, we conclude that

$$
\begin{aligned}
& \int_{\tau}^{T_{*}} t^{2}\left\|\sqrt{\rho} u_{t t t}\right\|_{L^{2}}^{2} d t+t^{2}\left\|\nabla u_{t t}(t)\right\|_{L^{2}}^{2} \\
\leq & C+C \tau^{2}\left\|\nabla u_{t t}(\tau)\right\|_{L^{2}}^{2}+\left|T_{*}^{2} f\left(T_{*}\right)\right|+\left|\tau^{2} f(\tau)\right|+C \int_{\tau}^{T_{*}} t|f(t)| d t .
\end{aligned}
$$

It is easy to show that

$$
t|f(t)| \leq C(\varepsilon)+\varepsilon t^{2}\left\|\nabla u_{t t}\right\|_{L^{2}}^{2}, \text { for any } t \in\left[0, T_{*}\right) .
$$


Therefore, recalling that

$$
\int_{0}^{T_{*}} t\left\|\nabla u_{t t}(t)\right\|_{L^{2}}^{2} d t \leq C
$$

and

$$
\tau_{k}^{2}\left\|\nabla u_{t t}\left(\tau_{k}\right)\right\|_{L^{2}}^{2} \rightarrow 0 \text { for some sequence }\left\{\tau_{k}\right\} \text { with } \tau_{k} \rightarrow 0,
$$

we deduce from (3.45) that

$$
\int_{0}^{T_{*}} t^{2}\left\|\sqrt{\rho} u_{t t t}\right\|_{L^{2}}^{2} d t+t^{2}\left\|\nabla u_{t t}(t)\right\|_{L^{2}}^{2} \leq C .
$$

Combining (3.42) and (3.46), we complete the proof of Lemma 3.4.

LEMMA 3.5. The strong solution of (1.1)-(1.2) satisfies

$$
t u_{t} \in L^{\infty}\left(0, T_{*} ; H^{2}\right), t^{2} u_{t t} \in L^{2}\left(0, T_{*} ; H^{2}\right), t u \in L^{\infty}\left(0, T_{*} ; H^{4}\right) .
$$

Proof. Differentiating $(1.1)_{2}$ with respect to $t$, leads to

$$
\begin{aligned}
& \rho u_{t t}+\rho_{t} u_{t}+(\rho u \cdot \nabla u)_{t}+\nabla P_{t}-\operatorname{div}\left(2 \mu^{\prime} \rho_{t} \mathcal{D} u\right)-\nabla\left(\lambda^{\prime} \rho_{t} \operatorname{div} u\right) \\
= & \operatorname{div}\left(2 \mu \mathcal{D} u_{t}\right)+\nabla\left(\lambda \operatorname{div} u_{t}\right) .
\end{aligned}
$$

This shows that $t u_{t}$ satisfies the following elliptic equation:

$$
\left\{\begin{array}{l}
\operatorname{div}\left(2 \mu t \mathcal{D} u_{t}\right)+\nabla\left(\lambda \operatorname{div}\left(t u_{t}\right)\right)=F_{1}, \\
\left(t u_{t}\right)(x, t)=0, x \in \partial \Omega, t \in\left(0, T_{*}\right),
\end{array}\right.
$$

where

$$
F_{1}=t \rho u_{t t}+t \rho_{t} u_{t}+t(\rho u \cdot \nabla u)_{t}+t \nabla P_{t}-2 t \operatorname{div}\left(\mu^{\prime} \rho_{t} \cdot \mathcal{D} u\right)-t \nabla\left(\lambda^{\prime} \rho_{t} \operatorname{div} u\right) .
$$

Note that (3.36) and Lemma 3.4 imply $F_{1} \in L^{\infty}\left(0, T_{*} ; L^{2}\right)$. Then it follows from elliptic system theory that

$$
\left\|\nabla^{2}\left(t u_{t}\right)\right\|_{L^{2}} \leq\left(\left\|F_{1}\right\|_{L^{2}}+\left\|2 \mu^{\prime} \nabla \rho \cdot \mathcal{D} u_{t}+\lambda^{\prime} \nabla \rho \operatorname{div} u_{t}\right\|_{L^{2}}\right)
$$

and

$$
\nabla^{2}\left(t u_{t}\right) \in L^{\infty}\left(0, T_{*} ; L^{2}\right)
$$

From $(1.1)_{2}, t^{2} u_{t t}$ satisfies

$$
\left\{\begin{array}{l}
\operatorname{div}\left(2 \mu t^{2} \mathcal{D} u_{t t}\right)+\nabla\left(\lambda \operatorname{div}\left(t^{2} u_{t t}\right)\right)=F_{2} \\
\left(t^{2} u_{t t}\right)(x, t)=0, \quad x \in \partial \Omega, t \in\left(0, T_{*}\right)
\end{array}\right.
$$

where

$$
\begin{array}{r}
F_{2}=t^{2}\left(\rho u_{t t t}+\nabla P_{t t}+\rho(u \cdot \nabla u)_{t t}+2 \rho_{t}\left(u \cdot \nabla u+u_{t}\right)_{t}+\rho_{t t}\left(u \cdot \nabla u+u_{t t}\right)\right. \\
\left.-\operatorname{div}\left(2 \mu_{t t} \mathcal{D} u\right)-\nabla\left(\lambda_{t t} \operatorname{div} u\right)-\operatorname{div}\left(4 \mu_{t} \mathcal{D} u_{t}\right)-\nabla\left(2 \lambda_{t} \operatorname{div} u_{t}\right)\right) .
\end{array}
$$


Note that (3.36), Lemma 3.4, and (3.48) imply $F_{2} \in L^{2}\left(0, T_{*} ; L^{2}\right)$. Then it follows from the elliptic system theory that

$$
\left\|\nabla^{2}\left(t^{2} u_{t t}\right)\right\|_{L^{2}} \leq C\left(\left\|F_{2}\right\|_{L^{2}}+\left\|2 \mu^{\prime} \nabla \rho \cdot \mathcal{D} u_{t t}+\lambda^{\prime} \nabla \rho \operatorname{div} u_{t t}\right\|_{L^{2}}\right)
$$

and

$$
\nabla^{2}\left(t^{2} u_{t t}\right) \in L^{2}\left(0, T_{*} ; L^{2}\right)
$$

Differentiating $(1.1)_{2}$ with respect to $x_{i}$ and $x_{j}$ and multiplying $t^{2}$ leads to

$$
\operatorname{div}\left(2 \mu t^{2} \mathcal{D} \partial_{i} \partial_{j} u\right)+\nabla\left(\lambda \operatorname{div}\left(t^{2} \partial_{i} \partial_{j} u\right)\right)=F_{3},
$$

where

$$
\begin{aligned}
F_{3}= & t^{2} \partial_{i} \partial_{j}\left\{\rho u_{t}+\rho u \cdot u+\nabla P(\rho)\right\}-\operatorname{div}\left(2 \partial_{i} \partial_{j} \mu t^{2} \mathcal{D} u\right)-\nabla\left(\partial_{i} \partial_{j} \lambda \operatorname{div}\left(t^{2} u\right)\right) \\
& -\operatorname{div}\left(2 \partial_{i} \mu t^{2} \mathcal{D} \partial_{j} u\right)-\operatorname{div}\left(2 \partial_{j} \mu t^{2} \mathcal{D} \partial_{i} u\right)-\nabla\left(\partial_{i} \lambda \operatorname{div}\left(t^{2} \partial_{j} u\right)\right)-\nabla\left(\partial_{j} \lambda \operatorname{div}\left(t^{2} \partial_{i} u\right)\right) .
\end{aligned}
$$

Note that (3.36), Lemma 3.4, (3.48), (3.49), and Sobolev's inequality imply $F_{3} \in$ $L^{\infty}\left(0, T_{*} ; L^{2}\right)$.

Then it follows from elliptic system theory that

$$
\left\|\nabla^{4}\left(t^{2} u\right)\right\|_{L^{2}} \leq C\left(\left\|F_{3}\right\|_{L^{2}}+\left\|2 \mu^{\prime} \nabla \rho \cdot \mathcal{D} \partial_{i} \partial_{j} u+\lambda^{\prime} \nabla \rho \operatorname{div} \partial_{i} \partial_{j} u\right\|_{L^{2}}\right)
$$

and

$$
\nabla^{4}\left(t^{2} u\right) \in L^{\infty}\left(0, T_{*} ; L^{2}\right) .
$$

Combining (3.48), (3.49), and (3.50), we complete the proof of Lemma 3.5.

Combining lemmas 3.1-3.5, Theorem 1.1 is proved.

\section{Proofs of Theorem 1.2 and Theorem 1.3}

Proof of Theorem 1.2. We use the result of Theorem 1.1 to prove Theorem 1.2 . that

By virtue of the assumptions of Theorem 1.2, there exist a constant $\delta>0$ such

$$
\inf _{x \in \Omega} \rho>\delta
$$

Set

$$
\mu_{\delta}(\rho)= \begin{cases}A_{1} \rho^{\alpha}, & \text { if } \rho>\delta / 4, \\ A_{1}\left(\frac{\delta}{4}\right)^{\alpha}+A_{1} \alpha\left(\frac{\delta}{4}\right)^{\alpha-1}(\rho-\delta / 4)+A_{1} \frac{\alpha(\alpha-1)}{2}\left(\frac{\delta}{4}\right)^{\alpha-2}(\rho-\delta / 4)^{2} & \\ +A_{1} \frac{\alpha(\alpha-1)(\alpha-2)}{6}\left(\frac{\delta}{4}\right)^{\alpha-3}(\rho-\delta / 4)^{3}+C(\rho-\delta / 4)^{4}, & \text { if } \rho \leq \delta / 4 .\end{cases}
$$

Clearly if we choose $\mathrm{C}$ large enough, we have

$$
\mu_{\delta}(\cdot) \in C^{3}[0, \infty) \text { and } \mu_{\delta} \geq C(\delta)>0,\left\|\frac{\partial^{j} \mu_{\delta}}{d \rho^{j}}\right\|_{L^{\infty}} \leq C_{1}(\delta), j=1,2,3 .
$$

We consider the following problem:

$$
\left\{\begin{array}{l}
\rho_{t}+\operatorname{div}(\rho u)=0 \\
(\rho u)_{t}+\operatorname{div}(\rho u \otimes u)+L u+\nabla P(\rho)=0 \\
L u=-\operatorname{div}\left(2 \mu_{\delta} \mathcal{D} u\right)-\nabla(\lambda \operatorname{div} u)
\end{array}\right.
$$


and the boundary conditions

$$
\begin{cases}\left.(\rho, u)\right|_{t=0}= & \left(\rho_{0}, u_{0}\right), \text { in } \Omega \\ u=0, & \text { on }(0, T) \times \partial \Omega .\end{cases}
$$

By virtue of Theorem 1.1, (4.1)-(4.2) has a local solution $\left(\rho_{\delta}, u_{\delta}\right)$ which satisfies

$$
\sup _{0 \leq t \leq T}\left\|u_{\delta}\right\|_{H^{3}} \leq C(\delta)
$$

Note that

$$
\rho_{\delta}(t, x)=\rho_{0}(U(0, t, x)) \exp \left(-\int_{0}^{t} \operatorname{div} u_{\delta}(s, U(s, t, x)) d s\right)
$$

where $U=U(t, s, x)$ is the solution to

$$
\left\{\begin{array}{l}
\frac{\partial}{\partial t} U(t, s, x)=u_{\delta}(t, U(t, s, x)), \quad 0 \leq t \leq T \\
U(s, s, x)=x, \quad 0 \leq s \leq T, \quad x \in \Omega
\end{array}\right.
$$

Hence there is a time $T^{*}>0, T^{*} \leq T$ such that

$$
\rho_{\delta} \geq \frac{\delta}{2}
$$

and this implies $\left(\rho_{\delta}, u_{\delta}\right)$ is a classical solution of (1.1)-(1.2). Theorem 1.2 is proved.

Proof of Theorem 1.3. We define $\phi^{R}(x)=\phi(x / R)$, where $\phi \in C_{0}^{\infty}\left(B_{1}\right)$ is a smooth cut-off function such that $\phi=1$ in $B_{1 / 2}$. Denote $\rho_{0}^{R}=\rho_{0}+R^{-3}, g^{R}=\phi^{R} g$. Let $u_{0 \delta} \in H_{0}^{1} \cap H^{3}$ be a unique solution to the following elliptic boundary value problem:

$$
\begin{aligned}
& L_{R} u_{0}^{R}=-\operatorname{div}\left(2 \mu\left(\rho_{0}^{R}\right) \mathcal{D} u_{0}^{R}\right)-\nabla\left(\lambda\left(\rho_{0}^{R}\right) \operatorname{div} u_{0}^{R}\right)=F_{0}^{R}, \text { in } B_{R}, \\
& \text { and } u_{0}^{R}=0, \text { on } \partial B_{R},
\end{aligned}
$$

where

$$
F_{0}^{R}=-\nabla P\left(\rho_{0}^{R}\right)+\rho_{0}^{R} g^{R}, \text { and } F_{0}=-\nabla P\left(\rho_{0}\right)+\rho_{0} g .
$$

We will show that for any $R_{0}>0$, there exists a sequence $R_{j}, R_{j} \rightarrow \infty$ such that

$$
\left\{\begin{array}{l}
u_{0}^{R_{j}} \rightarrow u_{0}, \text { in } H^{2}\left(B_{R_{0}}\right) \text { as } R_{j} \rightarrow \infty \\
D^{3} u_{0}^{R_{j}} \rightarrow D^{3} u_{0}, \text { in } L^{2}\left(B_{R_{0}}\right) \text { as } R_{j} \rightarrow \infty \\
\text { and }\left\|D^{3} u_{0}^{R}\right\|_{L^{2}\left(B_{R_{0}}\right)} \leq C\left\|D^{3} u_{0}\right\|_{L^{2}}+C
\end{array}\right.
$$

where $\mathrm{C}$ is a constant independent of $R_{j}, R_{0}$. According to the theory of elliptic systems, (4.3) has a unique solution $u_{0}^{R} \in H^{3}\left(B_{R}\right)$, and

$$
\begin{aligned}
& \int_{B_{R}}\left(2 \mu\left(\rho_{0}^{R}\right)\left|\nabla u_{0}^{R}\right|^{2}+\lambda\left(\rho_{0}^{R}\right)\left(\operatorname{div} u_{0}^{R}\right)^{2}\right) d x \\
= & \int_{B_{R}}\left(2 \mu\left(\rho_{0}\right) \nabla u_{0} \cdot \nabla u_{0}^{R}+\lambda\left(\rho_{0}\right) \operatorname{div} u_{0} \operatorname{div} u_{0}^{R}\right) d x+\int_{B_{R}}\left(F_{0}^{R}-F_{0}\right) \cdot u_{0}^{R} d x .
\end{aligned}
$$


Note that

$$
\begin{aligned}
& \int_{B_{R}}\left(F_{0}^{R}-F_{0}\right) \cdot u_{0}^{R} d x \\
\leq & \int_{B_{R}}\left|P\left(\rho_{0}^{R}\right)-P\left(\rho_{0}\right)\right|\left|\nabla u_{0}^{R}\right| d x+R^{-3} \int_{B_{R}}\left|g u_{0}^{R}\right| d x+\int_{B_{R}} \rho_{0}\left(\phi^{R}-1\right) g \cdot u_{0}^{R} d x .
\end{aligned}
$$

Using Poincaré's inequality, we have

$$
\begin{aligned}
& R^{-3} \int_{B_{R}}\left|g u_{0}^{R}\right| d x \leq R^{-3}\left(\|g\|_{L^{2}}^{2}+\left\|u_{0}^{R}\right\|_{L^{2}}^{2}\right) \leq C+C R^{-1}\left\|\nabla u_{0}^{R}\right\|_{L^{2}}^{2}, \\
& \quad \int_{B_{R}} \rho_{0}\left(\phi^{R}-1\right) g \cdot u_{0}^{R} d x=\int_{B_{R}}\left(\phi^{R}-1\right)\left(L u_{0}+\nabla P\left(u_{0}\right)\right) \cdot u_{0}^{R} d x \\
& \leq C \int_{B_{R}}\left(\left|\nabla \phi^{R}\right|\left|u_{0}^{R}\right|+\left|\phi^{R}-1 \| \nabla u_{0}^{R}\right|\right)\left(\left|\nabla u_{0}\right|+P\left(\rho_{0}\right)\right) d x \\
& \leq \epsilon\left\|\nabla u_{0}^{R}\right\|_{L^{2}}^{2}+C(\epsilon),
\end{aligned}
$$

where $L u_{0}=-\operatorname{div}\left(2 \mu\left(\rho_{0}\right) \mathcal{D} u_{0}\right)-\nabla\left(\lambda\left(\rho_{0}\right) \operatorname{div} u_{0}\right)=-\nabla P\left(\rho_{0}\right)+\rho_{0} g=F_{0}$, Hence from (4.5)-(4.7) and Sobolev's inequality, it follows that

$$
\left\|\nabla u_{0}^{R}\right\|_{L^{2}\left(B_{R}\right)} \leq C \text { and }\left\|u_{0}^{R}\right\|_{L^{6}\left(B_{R}\right)} \leq C\left\|\nabla u_{0}^{R}\right\|_{L^{2}\left(B_{R}\right)} \leq C_{1} .
$$

According to the theory of elliptic systems,

$$
\left\|\nabla u_{0}^{R}\right\|_{H^{2}\left(B_{R}\right)} \leq C\left\|F_{0}^{R}\right\|_{H^{1}\left(B_{R}\right)}+C\left\|\nabla \rho_{0}\right\|_{H^{2}} \leq C_{2},
$$

where C, $C_{1}$, and $C_{2}$ are constants independent of $\mathrm{R}$ and $\nabla P\left(\rho_{0}^{R}\right) \rightarrow \nabla P\left(\rho_{0}\right)$ in $H^{1}$ is used. This means that for any $R_{0}>0$ there exists a sequence $R_{j}, R_{j} \rightarrow \infty$ such that $\left\{u_{0}^{R_{j}}\right\}$ converges strongly in $H^{2}\left(B_{R_{0}}\right)$ to a limit $\bar{u}_{0} \in H^{2}\left(B_{R_{0}}\right)$ and $\left\{D^{3} u_{0}^{R_{j}}\right\}$ converges weakly in $L^{2}\left(B_{R_{0}}\right)$ to $D^{3} \bar{u}_{0} \in L^{2}$. Thus there exists a subsequence of $\left\{u_{0}^{R_{j}}\right\}$ (still denoted by $\left.\left\{u_{0}^{R_{j}}\right\}\right)$ such that $u_{0}^{R}, D u_{0}^{R_{j}}, D^{2} u_{0}^{R_{j}} \rightarrow \bar{u}_{0}, D \bar{u}_{0}, D^{2} \bar{u}_{0}$ a.e. on $\mathbb{R}^{3}$. Letting $R_{j} \rightarrow \infty$ in (4.3), we get

$$
L \bar{u}_{0}=-\operatorname{div}\left(2 \mu\left(\rho_{0}\right) \mathcal{D} \bar{u}_{0}\right)-\nabla\left(\lambda\left(\rho_{0}\right) \operatorname{div} \bar{u}_{0}\right)=-\nabla P\left(\rho_{0}\right)+\rho_{0} g=F_{0}, \bar{u}_{0} \in D^{1} \cap D^{2}
$$

a.e. in $\mathbb{R}^{3}$. Note that $L u_{0}=F_{0}$. The uniqueness of solution of elliptic problem implies $\bar{u}_{0}=u_{0}$. Hence (4.4) holds.

We now consider the first boundary value problem of (1.1) in a ball $B_{R} \subseteq \mathbb{R}^{3}$. The boundary conditions are as follows:

$$
\left\{\begin{array}{lr}
\left.(\rho, u)\right|_{t=0}=\left(\rho_{0}^{R}, u_{0}^{R}\right)=\left(\rho_{0}+R^{-3}, u_{0}^{R}\right), \text { in } B_{R}, \\
u=0, & \text { on } \quad(0, T) \times \partial B_{R} .
\end{array}\right.
$$

By virtue of Theorem 1.1, there is a time $T>0$, such that (1.1) and (4.9) has a unique solution $\left(\rho^{R}, u^{R}\right)$ satisfying (1.7). Note that the time $\mathrm{T}$ depends only on $\left\|\sqrt{\rho_{0}^{R}} g^{R}\right\|_{L^{2}},\left\|\nabla g^{R}\right\|_{L^{2}},\left\|\nabla u_{0}^{R}\right\|_{H^{2}},\left\|\rho_{0}^{R}\right\|_{L^{2}},\left\|P\left(\rho_{0}^{R}\right)\right\|_{H^{3}},\left\|\nabla \rho_{0}^{R}\right\|_{H^{3}}$. From (4.4) and $g^{R} \rightarrow g$ in $D^{1}, \sqrt{\rho_{0}^{R}} g^{R} \rightarrow \sqrt{\rho_{0}} g$ in $L^{2}$ as $R \rightarrow \infty$, it is easy to show that there is a time $T_{*}>0$ such that for any $R>0,(1.1)$ and (4.9) has a solution $\left(\rho^{R}, u^{R}\right)$ in $\left(0, T_{*}\right)$. 
According to the estimates (3.33)-(3.35), $\left(\rho^{R}, u^{R}\right)$ satisfies the following estimates, which are similar to $(3.33)-(3.35)$ :

$$
\begin{aligned}
& \sup _{0 \leq t \leq T_{*}}\left(\left\|\rho^{R}\right\|_{H^{3}\left(B_{R}\right)}^{2}+\left\|P\left(\rho^{R}\right)\right\|_{H^{3}\left(B_{R}\right)}^{2}+\left\|u^{R}\right\|_{D^{1} \cap D^{3}\left(B_{R}\right)}^{2}+\left\|\sqrt{\rho^{R}} u_{t}^{R}\right\|_{L^{2}\left(B_{R}\right)}^{2}\right. \\
& \left.\quad+\left\|u_{t}^{R}\right\|_{D^{1}\left(B_{R}\right)}^{2}\right)+\int_{0}^{T_{*}}\left(\left\|u^{R}\right\|_{D^{4}\left(B_{R}\right)}^{2}+\left\|u_{t}^{R}\right\|_{D^{2}\left(B_{R}\right)}^{2}+\left\|\sqrt{\rho^{R}} u_{t t}^{R}\right\|_{L^{2}\left(B_{R}\right)}^{2}\right) d t \leq C .
\end{aligned}
$$

Moreover, (4.10) and (1.1) imply

$$
\begin{aligned}
\sup _{0 \leq t \leq T_{*}}\left(\left\|\rho_{t}^{R}\right\|_{H^{2}\left(B_{R}\right)}^{2}+\left\|P_{t}\left(\rho^{R}\right)\right\|_{H^{2}\left(B_{R}\right)}^{2}+\left\|\rho_{t t}^{R}\right\|_{L^{2}\left(B_{R}\right)}^{2}+\left\|P_{t t}\left(\rho^{R}\right)\right\|_{L^{2}\left(B_{R}\right)}^{2}\right) & \\
+ & \left.\int_{0}^{T_{*}}\left(\left\|\nabla \rho_{t t}^{R}\right\|_{L^{2}\left(B_{R}\right)}^{2}+\left\|\nabla P_{t t}\left(\rho^{R}\right)\right\|_{L^{2}\left(B_{R}\right)}^{2}\right) d t \leq \mathbb{C} 4.11\right)
\end{aligned}
$$

where the constant $\mathrm{C}$ does not depend on $R$.

Hence the solution of the Cauchy problem (1.1), (1.8)-(1.10) can be obtained as the limit of a sequence of solutions to (1.1), (4.9) by choosing $R_{0}=R_{j} R_{j} \rightarrow \infty$ as $j \rightarrow \infty$. We omit the details of the argument.

Acknowledgments. The first author was supported by National Natural Science Foundation of China (Grant No. 11001090) and the Fundamental Research Funds for the Central Universities (Grant No. 11QZR16). The second author was supported by National Natural Science Foundation of China (Grant No. 10971171).

\section{REFERENCES}

[1] D. Bresch, B. Desjardins, and D. Gerard-Varet, On compressible Navier-Stokes equations with density dependent viscosities in bounded domains, J. Math. Pures Appl., 87, 227-235, 2007.

[2] Y.Z. Chen and L.C. Wu, Second Order Elliptic Equation and Elliptic System, Amer. Math. Soc., 1998.

[3] Y. Cho, H.J. Choe, and H. Kim, Unique solvability of the initial boundary value problems for compressible viscous fluid, J. Math. Pures Appl., 83, 243-275, 2004.

[4] Y. Cho and H. Kim, On classical solutions of the compressible Navier-Stokes equations with nonnegative initial densities, Manuscript Math., 120, 91-129, 2006.

[5] H.J. Choe and H. Kim, Strong solutions of the Navier-Stokes equations for isentropic compressible fluids, J. Diff. Eqs., 190, 504-523, 2003.

[6] D.Y. Fang and T. Zhang, A note on compressible Navier-Stokes equations with vacuum state in one dimension, Nonlinear Anal., 58, 719-731, 2004.

[7] E. Feireisl, A. Novotny, and H. Petzeltová, On the existence of globally defined weak solutions to the Navier-Stokes equations, J. Math. Fluid Mech., 3(4), 358-392, 2001.

[8] Z.H. Guo, S. Jiang, and F. Xie, Global existence and asymptotic behavior of weak solutions to the $1 D$ compressible Navier-Stokes equations with degenerate viscosity coefficient, Asym. Anal., 60, 101-123, 2008.

[9] Z.H. Guo and C.J. Zhu, Global weak solutions and asymptotic behavior to $1 D$ compressible Navier-Stokes equations with density-dependent viscosity and vacuum, J. Diff. Eqs., 248, 2768-2799, 2010

[10] H. Grad, Asymptotic theory of the Boltzmann equation II, in: J. Laurmann (Ed.), Rarefied Gas Dynamics, Academic Press, New York, 1, 1963.

[11] D. Hoff, Global existence for 1D, compressible, isentropic Navier-Stokes equations with large initial data, Trans. Amer. Math. Soc., 303(1), 169-181, 1987.

[12] D. Hoff, Global solutions of the Navier-Stokes equations for multidimensional compressible flow with discontinuous initial data, J. Diff. Eqs., 120(1), 215-254, 1995.

[13] D. Hoff, Strong convergence to global solutions for multidimensional flow of compressible, viscous fluids with polytropic equations of state and discontinuous initial data, Arch. Rat. Mech. Anal., 132, 1-14, 1995. 
[14] X.D. Huang, J. Li, and Z.P. Xin, Global well-posedness of classical solutions with large oscillations and vacuum to the three-dimensional isentropic compressible Navier-Stokes equations, Commun. Pure Appl. Math., 65(4), 549-585, 2012.

[15] H. Ito, Extended Korn's inequality and the associated best possible constant, J. Elasticity, 24, 43-78, 1990.

[16] S. Jiang, Z.P. Xin, and P. Zhang, Global weak solutions to $1 D$ compressible isentropic NavierStokes equations with density-dependent viscosity, Meth. Appl. Anal., 12(3), 239-252, 2005.

[17] M. Kawashita, On global solutions of Cauchy problems for compressible Navier-Stokes equations, Nonlinear Anal., 48, 1087-1105, 2002.

[18] A.V. Kazhikhov and V.V. Shelukhin, Unique global solution with respect to time of initialboundary value problems for one-dimensional equations of a viscous gas, Prikl. Mat. Meh., 41, 282-291, 1977.

[19] O.A. Ladyzenskaja, V.A. Solonnikov, and N.N. Uraltseva, Linear and Quasilinear Equations of Parabolic Type, Amer. Math. Soc., 1968.

[20] O.A. Ladyzenskaja and N.N. Uraltseva, Linear and Quasilinear Elliptic Equations, Academic Press, 1978.

[21] P.L. Lions, Mathematical Topics in Fluid Mechanics, Compressible Models, New York, Oxford University Press, 2, 1998.

[22] T.P. Liu, Z.P. Xin, and T. Yang, Vacuum states of compressible flow, Disc. Cont. Dyn. Syst., 4(1), 1-32, 1998.

[23] T. Makino, On a local existence theorem for the evolution equations of gaseous stars, in: Patterns and Wave-Qualitative Analysis of Nonlinear Differential Equations, North-Holland, 459-479, 1986.

[24] A. Matsumura and T. Nishida, The initial value problem for the equations of motion of viscous and heat-conductive gases, J. Math. Kyoto Univ., 20(1), 67-104, 1980.

[25] A. Mellet and A. Vasseur, On the barotropic compressible Navier-Stokes equations, Commun. Part. Diff. Eqs., 32(3), 431-452, 2007.

[26] J. Nash, Le probleme de Cauchy pour les équations différentielles d'un fluide général, Bull. Soc. Math. France, 90, 487-497, 1962.

[27] D. Serre, Solutions faibles globales des équations de Navier-Stokes pour un fluide compressible, C.R. Acad. Sci. Paris Sér. I Math., 303, 639-642, 1986.

[28] D. Serre, Sur l'équation monodimensionnelle dún fluide visqueux, compressible et conducteur de chaleur, C.R. Acad. Sci. Paris Sér. I Math., 303, 703-706, 1986.

[29] J. Serrin, On the uniqueness of compressible fluid motion, Arch. Rat. Mech. Anal., 3, 271-288, 1959.

[30] A. Valli, An existence theorem for compressible viscous fluids, Ann. Math. Pura. Appl., 130(1), 197-213, 1982.

[31] S.W. Vong, T. Yang, and C.J. Zhu, Compressible Navier-Stokes equations with degenerate viscosity coefficient and vacuum (II), J. Diff. Eqs., 192, 475-501, 2003.

[32] T. Yang and H.J. Zhao, A vacuum problem for the one-dimensional compressible Navier-Stokes equations with density-dependent viscosity, J. Diff. Eqs., 181(1), 163-184, 2002.

[33] T. Yang and C.J. Zhu, Compressible Navier-Stokes equations with degenerate viscosity coeffcient and vacuum, Commun. Math. Phys., 230, 329-363, 2002.

[34] T. Zhang and D.Y. Fang, Global behavior of spherically symmetric Navier-Stokes equations with density-dependent viscosity, J. Diff. Eqs., 236, 293-341, 2007. 